\title{
Choosing the right packing in millipacked bed reactors under single phase gas flow
}

\author{
Vittorio Petrazzuoli a,b, Matthieu Rolland ${ }^{a}$, Adrien Mekki-Berrada ${ }^{a}$, \\ Olivier Said-Aizpuru ${ }^{\text {a }}$, Yves Schuurman ${ }^{\text {b,* }}$ \\ a IFP Energies nouvelles, Rond-point de l'échangeur de Solaize, BP 3, 69360 Solaize, France \\ ${ }^{\mathrm{b}}$ Univ Lyon, Université Claude Bernard Lyon 1, CNRS, IRCELYON-UMR5256, 2 Avenue Albert Einstein F-69626, Villeurbanne, France
}

\begin{abstract}
Packed bed reactors with diameters below $1 \mathrm{~cm}$, millipacked beds hereafter, are often used to test catalysts. The prospect of using these reactors for catalytic kinetic measurements is quite attractive, but it requires a better knowledge of the flow characteristics of these reactors. This study focuses on the main hydrodynamic features of millipacked bed reactors in single phase gas flow. The effects of the bed length, gas velocity, reactor/particle diameter ratio $(\delta)$ and the use of fine inert powder as porosity filler are investigated via residence time distribution measurements and reactive tests. When using spherical particles, for values of reactor/particle diameter ratios between $1<\delta<3$, the behavior of the Peclet number, Pe, is not monotonous. Lower values of the Pe number for specific values of $\delta$ corresponding to large void spaces between the particles and/or between the particles and the walls, have been observed. For values of $\delta>3$ higher Pe numbers are observed, in particular at high gas velocities, due to the transition to a more uniform packing where the walls are no longer the major constraint. When using cylindrical particles, higher dispersion is observed when the cylinders align on each other along the reactor axis, as compared to when they are randomly arranged in the reactor. A criterion to calculate the maximum conversion that can be achieved neglecting dispersion effects has been proposed, highlighting the situations where it has to be used with caution. The dispersion in these reactors can often be neglected. In case the criterion is not fulfilled, filling the porosity of the beds with powder reduces the dispersion. Using porosity fillers in reactors with internal diameters between 2 and $4 \mathrm{~mm}$ showed an improvement of the conversions for $n$-heptane reforming and methylcyclohexane dehydrogenation, which is attributed to external mass transfer improvement. Fine and spherical powders fill better the porosity and yield lower dispersion and higher mass transfer.
\end{abstract}

\footnotetext{
* Corresponding author.

E-mail address: yves.schuurman@ircelyon.univ-lyon1.fr (Y. Schuurman).
} 


\section{Nomenclature}

\section{Roman letters}

C: $\quad$ concentration $\left[\mathrm{mol} / \mathrm{m}^{3}\right]$

$\mathrm{d}_{32}: \quad$ Sauter mean diameter $[\mathrm{m}]$

$\mathrm{D}_{\mathrm{ax}}$ : axial dispersion coefficient $\left[\mathrm{m}^{2} / \mathrm{s}\right]$

$\mathrm{D}_{\text {cyl }}$ : cylindrical particle diameter [m]

$\mathrm{D}_{\mathrm{m}}$ : molecular diffusivity $\left[\mathrm{m}^{2} / \mathrm{s}\right]$

$\mathrm{d}_{\mathrm{p}}$ : particles (equivalent) diameter $[\mathrm{m}]$

$\mathrm{D}_{\mathrm{r}}$ : $\quad$ reactor internal diameter $[\mathrm{m}]$

$\mathrm{D}_{\mathrm{T}}$ : $\quad$ transverse dispersion coefficient $\left[\mathrm{m}^{2} / \mathrm{s}\right]$

$\mathrm{E}_{\mathrm{a}}$ : $\quad$ activation energy $[\mathrm{J} / \mathrm{mol}]$

GHSV: gas hourly space velocity $\left[\mathrm{m}_{\text {gas }}^{3} /\left(\mathrm{m}_{\text {cat }}^{3} * \mathrm{~h}\right)\right]$

$\mathrm{K}_{\mathrm{gs}}$ : $\quad$ gas-solid mass transfer coefficient [m/s]

L: $\quad$ bed length $[\mathrm{m}]$

$\mathrm{L}_{\text {cyl }}$ : cylindrical particles length $[\mathrm{m}]$

LHSV: liquid hourly space velocity $\left[\mathrm{m}_{\mathrm{liq}}^{3} /\left(\mathrm{m}_{\text {cat }}^{3}{ }^{*} \mathrm{~h}\right)\right]$

n: reaction order

Q: $\quad$ gas flow rate $\left[\mathrm{m}^{3} / \mathrm{s}\right]$

R: $\quad$ gas constant $8.314[\mathrm{~J} /(\mathrm{K} \mathrm{mol})]$

$\mathrm{r}$ Volumetric reaction rate $\left[\mathrm{mol} /\left(\mathrm{m}^{3} \mathrm{~s}\right)\right]$

T: $\quad$ temperature $\left[\mathrm{K}\right.$ or $\left.{ }^{\circ} \mathrm{C}\right]$

$\mathrm{t}: \quad$ time [s]

$\mathrm{U} \quad$ inlet superficial velocity $[\mathrm{m} / \mathrm{s}]$

$\mathrm{u}_{\mathrm{i}}: \mathrm{u} / \varepsilon \quad$ inlet interstitial velocity $[\mathrm{m} / \mathrm{s}]$

$\mathrm{V}_{\mathrm{r}}$ : $\quad$ volume of the reactor $\left[\mathrm{m}^{3}\right]$

WHSV: reactant hourly velocity per catalyst mass unit [ $g_{\text {reactant }} /$ $\left.\left(\mathrm{h}^{*} \mathrm{~g}_{\text {cat }}\right)\right] \approx 1 / \mathrm{h}$

$\mathrm{X}_{\text {filler, } \mathrm{i}}$ : conversion of the reactor packed with the porosity filler $\mathrm{i}$

$\mathrm{X}_{\text {no_filler: }}$ conversion of the reactor packed without porosity fillers

$\mathrm{z} \quad$ Axial coordinate along the reactor axis [m]
Greek letters

$\alpha_{\mathrm{w}}$ : $\quad$ wall heat transfer coefficient $\left[\mathrm{W} /\left(\mathrm{m}^{2} \mathrm{~K}\right)\right]$

$\gamma-\lambda_{0}-\lambda_{1}-\lambda_{2}-\beta$ : parameters for the Bo correlation

$\delta: \frac{D_{r}}{d_{p}} \quad$ reactor/particle diameter ratio (beads)

$\delta^{\prime}: \mathrm{D}_{\mathrm{r}} \mathrm{d}_{\mathrm{cyl}} \quad$ reactor/particle diameter ratio (cylinders)

$\Delta \mathrm{H}^{\mathrm{o}}: \quad$ standard reaction enthalpy $[\mathrm{J} / \mathrm{mol}]$

$\Delta \mathrm{T}_{\text {rad }}$ : temperature variation in the radial direction [ $\mathrm{K}$ or ${ }^{\circ} \mathrm{C}$ ]

$\Delta \mathrm{X}: \quad$ conversion difference between reactors with and without porosity filler

$\Delta \mathrm{X}_{\mathrm{p}}$ : conversion difference between reactors packed with same size ZirBlast ${ }^{\circledR}$ and SiC

$\varepsilon: \quad$ void fraction of the reactor

$\mu$ : $\quad$ fluid viscosity [Pa s]

$\lambda: \quad$ thermal conductivity $[\mathrm{W} /(\mathrm{m} \mathrm{K})]$

$\rho: \quad$ fluid density $\left[\mathrm{kg} / \mathrm{m}^{3}\right]$

$\tau: \quad$ tortuosity factor of the packed bed

$\Phi: \quad$ pellet sphericity

$\psi: \frac{L_{\mathrm{cyl}}}{\mathrm{D}_{\mathrm{r}}} \quad$ cylinder length/reactor diameter ratio

Dimensionless numbers

Bo: $\frac{\mathrm{u}_{\mathrm{i}} * \mathrm{~d}_{\mathrm{p}}}{\mathrm{D}_{\mathrm{ax}}}$ bodenstein number

Pe: $\frac{\mathrm{u}_{\mathrm{i}} * \mathrm{~L}}{\mathrm{D}_{\mathrm{ax}}} \quad$ reactor Peclet number

$\mathrm{Pe}_{\mathrm{m}}: \frac{\mathrm{u}_{\mathrm{i}} * \mathrm{~d}_{\mathrm{p}}}{\mathrm{D}_{\mathrm{m}}}$ molecular Peclet number

Re: $\frac{\rho * \mathrm{u}_{i} * \mathrm{~d}_{\mathrm{p}}}{\mu}$ Reynolds number

\section{Introduction}

The development cycle of a catalyst begins with a broad screening of many catalyst formulations, which is followed by a detailed investigation of a selection of the best candidates aiming at gathering sufficient knowledge to design an industrial process and guarantee its performance. The industrial catalyst development is preferably based on the final millimetric size catalyst pellet rather than the powder sample for several reasons. As impregnation may depend on catalyst size, especially when impregnation solutions are very viscous, all catalysts must be prepared with millimetric size pellets to avoid up-scaling the impregnation procedure from powder to pellet. Testing powders requires an additional step of crushing or grinding, which may introduce some variability (i.e. small changes in the powder size distribution, pollution of metallic parts, etc....). A powder catalyst may perform differently in terms of conversion and selectivity in presence of internal and/or external mass and heat transfer limitations so that a model coupling kinetic and mass and/or heat transport is necessary to predict the millimetric size catalyst performances (Sie, 1996), introducing more uncertainty in the process design. Due to the high financial stakes in the performance guarantees, the model validation on millimetric size catalysts will be anyway performed and it is much more efficient to perform only millimetric catalyst testing than both millimetric and powder testing. Experimental tests on pellets are thus mandatory.

Over decades, packed bed reactors for catalyst testing have faced a reduction in size and have now diameters commonly below $1 \mathrm{~cm}$, the limit being the catalyst pellet size $(\sim 1-3 \mathrm{~mm})$. The advantages are the reduction of the development costs and delays: less catalyst (Moulijn et al., 2003), lower amount of feed- stock and waste (Templis and Papayannakos, 2017; Zhang et al., 2017), better temperature control (higher surface to volume ratio) (Zhang et al., 2017), easier implementation of parallel reactor systems (Moonen et al., 2017) yielding more reactors per unit area and per man-hour and reduced safety issues (Moonen et al., 2017). The experimentation performed using parallel reactor systems is known as High Throughput Experimentation (HTE). Recent HTE systems offer 8 or more reactors in parallel for the cost of a single unit. The reactors share most of the essential functions like analyzers, mass flow controllers, utilities, furnaces, feedstock tanks or back-pressure controllers, leading to cheaper units with smaller footprints. An interesting consequence of HTE is the possibility of replication/determination of the statistical significance of the results with reasonable costs and delays.

Millipacked bed reactors like their predecessors are operated keeping the L- or G-HSV (Liquid or Gas Hourly Space Velocity) similar to those on an industrial scale. This leads to a reduction of gas and liquid superficial velocities by a factor of 10 to 100 compared to industrial reactors, which results in an increase in mass and heat transfer resistances, reactors deviating from plug-flow behavior and, for gas-liquid flows, in changes of the flow patterns, phase distributions, and catalyst wetting (Sie, 1996).

Additional risks in operating reactors on the order of millimeters of diameter are: accuracy of small catalyst mass measurement, sampling bias when using a very small number of pellets (Rolland and Fonte, 2015), accurate mass flow control (equipment are used in their lower operating range, often a splitter is necessary to achieve low flow rates), lower effluents amounts, which may be limiting for analysis and potential packing issues (local reactor porosities, grain size differences and orientation). 
As millipacked reactors produce a large amount of data cheaply, it is quite tempting to use these units for up-scaling purposes. These reactors are validated for catalyst screening, where the important information is the ranking and some indication of the performance gains; in this case it is admissible that the information depends on the reactor size. In these systems, the hydrodynamics is not really an issue as long as the ranking is correct and the uncertainties are under control. On the opposite, for upscaling tests, it is preferred that the information is independent of the reactor used. Any reactor model imperfection will add uncertainties in the kinetic model optimization, uncertainties that will propagate in the final industrial design. It is therefore preferred by process engineering that the experimental raw data can be used directly without any reactor model. The goal of this paper is to explore the hydrodynamics and mass transfer performances of millipacked bed reactors and give insight on when experimental kinetic results can be up-scaled without models.

The reactors used in HTE consist of packed bed reactors with diameters smaller than $1 \mathrm{~cm}$ and lengths that usually do not exceed the $20-30 \mathrm{~cm}$. As the catalyst particles are on the order of a few millimeters, a millipacked bed is usually operated with values of $\delta=D_{r} / d_{p}$ (reactor/particle diameter ratio) between 1 and 5 , possibly 10 , while traditional packed beds have much higher value ( $>1000$ for industrial packed beds). In millipacked beds, the ratio $\delta=D_{r} / d_{p}$ governs the disposition of the particles in the reactor (example in Fig. 1 for beads). For large values of $\delta$ there are few preferential paths and dead-zones due to the stochastic position of the particles at the center of the bed and the higher packing density (Chu and Ng, 1989; McGeary, 1961), while for the low $\delta$ values occurring in millipacked beds the global void fraction is higher and radially non-uniform (Mueller, 1992) and preferential paths near the walls are important enough to drive most of the flow.

Although the hydrodynamics in gas-solid fixed beds has been measured and correlated extensively in large reactors, the literature for millipacked beds is significantly less abundant. The packed bed reactor hydrodynamics is usually well described by the axial dispersion model. This model assumes some degree of dispersion, a quantity physically analog to the diffusion of molecules, but augmented by the mixing induced by the flow. A common rule-ofthumb (Delgado, 2006) recommends values of $\delta$ higher than $\sim 15$, so that the radial porosity fluctuations near the walls have less impact on the average flow and the packing can be considered as

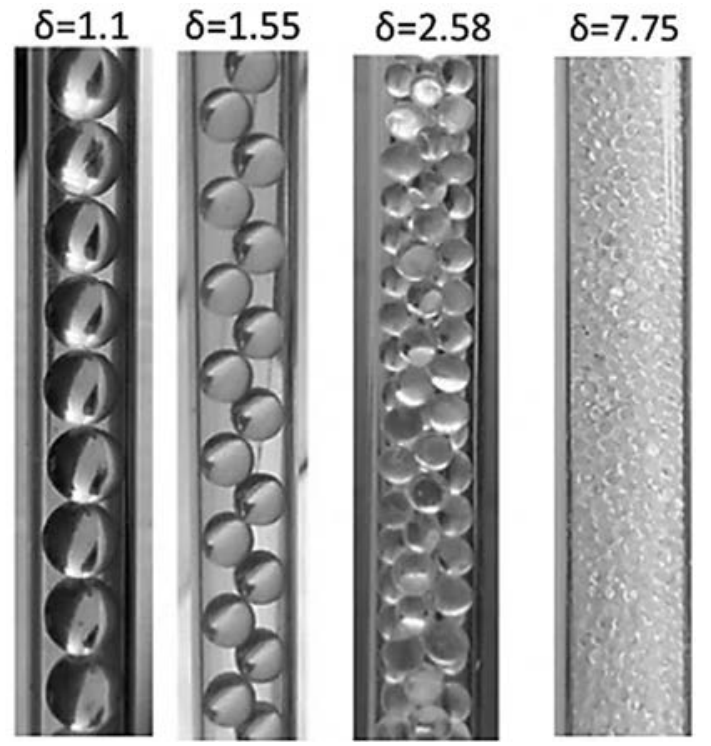

Fig. 1. Examples of spherical particles positioning against $\delta\left(D_{r} / d_{p}\right)$ value. uniform in the radial direction. In this case variations of the fluid velocity, porosity and axial dispersion coefficient in the radial direction can be neglected. Millipacked bed reactors do not respect this rule of thumb and another design criterion is required. A study from Johnson and Kapner using a large packed bed $\left(D_{r}=10 \mathrm{~cm}\right)$ (Johnson and Kapner, 1989) analyzed the effect of the $\delta$ values $(4<\delta<128)$ on the hydrodynamics, and concluded that the dispersion decreases with increasing $\delta$. Other studies from Šolcova and Schneider (Šolcova and Schneider, 2004), Scott et al. (Scott et al., 1974) and Hsiang and Haynes (Hsiang and Haynes, 1977) showed that low values of dispersion, comparable to larger packed beds, are achieved when $\delta$ is below 2 .

Another important aspect for correct reactor upscaling is the absence of mass and/or heat transfer limitations during kinetic measurements in lab-scale reactors. In absence of mass and/or heat transfer limitations, the kinetic regime, a change in the superficial velocity while keeping the L or G-HSV constant does not change the performances. This is checked by comparing the performances of beds with various lengths, long enough to neglect axial dispersion. Outside of this region, limitations must be accounted for, and external mass transfer in gas phase reactors can be evaluated experimentally by measuring apparent performances under conditions where the reaction rate is strongly governed by mass transfer. This process requires knowledge of the kinetics, which can only be deduced in absence of mass transfer limitations. To circumvent this loophole, the usual way is to measure the kinetics in another type of (unlimited) reactor. In this study, we opted to study the effect of the packing on mass transfer by consequently changing the hydrodynamics through the filling of the reactor's void space with fine powder. When the packing changes do not result in changes in the performances (while still having a response to temperature changes), we are likely to be in the kinetic regime. This approach will help to answer other pending questions about HTE reactors: should we use porosity filler? And what type (size and shape)?

In this experimental work we report the effect of different parameters on the hydrodynamics of millipacked bed reactors: bed length $(L)$, gas superficial velocity $(u)$, reactor diameter $\left(D_{r}\right)$, reactor/particle diameter ratio $(\delta)$ and the use of porosity filler. We then propose a criterion to estimate the maximum conversion that can be achieved neglecting dispersion effects. In a second part, the influence of porosity fillers on the performance of gas-solid reactions is investigated.

\section{Materials and methods}

In this part, we present the materials and methods for both the residence time distribution (RTD) measurement and the reactive tests.

\subsection{RTD measurements}

\subsubsection{Axial dispersion model}

The axial dispersion model is an adequate description of single phase flow in semi-infinite packed beds (Delgado, 2006) (L/ $\left.D_{r}>20\right)$. One objective is to assess if this model is suitable to describe the hydrodynamics of millipacked beds with small values of $\delta$ and $L / D_{r}<20$.

The model consists of a $1 \mathrm{D}$ mass balance equation corrected with a dispersion term (Eq. (1)):

$\frac{\partial C}{\partial t}=D_{a x} \frac{\partial^{2} C}{\partial z^{2}}-\frac{u}{\varepsilon} \frac{\partial C}{\partial z}$

in which $z$ is the axial coordinate along the bed, $C$ is the concentration of the gas phase species, $D_{a x}$ the axial dispersion coefficient, $u$ 
the superficial gas velocity, $\varepsilon$ the void fraction of the reactor and $t$ the clock time. Closed system boundary conditions have been chosen for inlet and outlet of the reactor.

The extent of dispersion is quantified in terms of the dimensionless Peclet $(\mathrm{Pe})$ and/or Bodenstein $(\mathrm{Bo})$ numbers:

$P e=\frac{u_{i} * L}{D_{a x}}$ Bo $=\frac{u_{i} * d_{p}}{D_{a x}}$

in which $u_{i}=u / \varepsilon$ is the gas interstitial velocity. The Peclet number compares the convection and the dispersion in the reactor at the reactor scale. The higher the value of the Peclet number, the better the reactor can be described by (ideal) plug-flow in which all the molecules have the same residence time in the reactor. The Bo number has the same meaning at the particle scale.

For cylinders, $d_{p}$ is the volume to area equivalent diameter (Eq. (3)), that is the diameter of a sphere that has the same ratio of volume to external area.

$d_{p}=\frac{6 \cdot \text { Volume }}{\text { Area }}=\frac{3 d_{c y l} L_{c y l}}{d_{c y l}+2 L_{c y l}}$

When using powder as porosity filler the particle diameter $d_{p}$ is the powder diameter, as it governs the hydrodynamics (Sie, 1996).

\subsubsection{RTD set-up}

We estimated the axial dispersion coefficient in the reactors by analyzing the transient concentration profiles of a tracer species after a step change. Blank profiles have been measured without the reactors, in order to take into account the dispersion coming from the upstream and downstream equipment in the future analysis (Šolcova and Schneider, 2004; Márquez et al., 2008). The tracer is helium in nitrogen with a step change from pure nitrogen to a $50-50 \%$ mixture (vol) of helium and nitrogen. The gas concentration is measured using mass spectrometry, a very common technique for gas phase RTD measurements (Klyushina et al., 2015).

The flow diagram of the RTD setup is shown in Fig. 2. A remote controlled 4-way valve (Vici valve from Valco Instruments Co. Inc.) switches the gas flow through the reactor from nitrogen (circuit B) to a mixture of $50 \%$ vol. nitrogen and $50 \%$ vol. helium (circuit A).
The gases are delivered by mass flow controllers (Brooks Instrument). A needle valve is used on the gas vent line to match the reactor pressure drop and eliminate the overshoot in the transient response. A mass spectrometer (INFICON) measures the temporal concentration profile of the tracer. The valve position change $(t=0)$ is recorded by the mass spectrometer with a time resolution of $85 \mathrm{~ms}$. At the end of the test, the temporal concentration profiles and the switching time are available on the same time track. The exact switching time will be the recorded time, with a time lag of maximum $85 \mathrm{~ms}$.

In this setup, the temporal concentration profiles also account for dispersion of the lines, the valve, and the MS inlet device. As their contribution to the dispersion does not depend on their order, we measure the system response without reactors and use these temporal profiles, called "blanks" afterwards, as the inlet profile for the axial dispersion model.

In order to improve the accuracy of the measurements, the mass flow controllers and mass spectrometer signal were regularly calibrated. The calibration has been performed using mixtures with known proportions of helium and nitrogen (from 0 to $50 \%$ in volume of helium) and recording the signal variations. Then, an interpolating curve is used to calculate the helium molar fraction according to the MS signal value. The mass to charge ratios analyzed for helium and nitrogen are respectively 4 and $28 \mathrm{~m} / z$.

RTD measurements without reactors have been repeated regularly during the test campaign and showed no significant deviation. All RTD measurements have been repeated 5 times (Fig. 3). The curves were found to be identical except for a time lag induced by the MS time resolution ( $85 \mathrm{~ms}$ ). In the model analysis, the median curve has been retained. The helium MS response signals presented some noise at high concentration levels.

\subsubsection{RTD data analysis}

The convection - dispersion Eq. (1) was solved in a numerical model used to propagate the inlet tracer concentration profiles through the reactor and predict the outlet tracer concentration profiles. The model uses finite differences and an adaptive

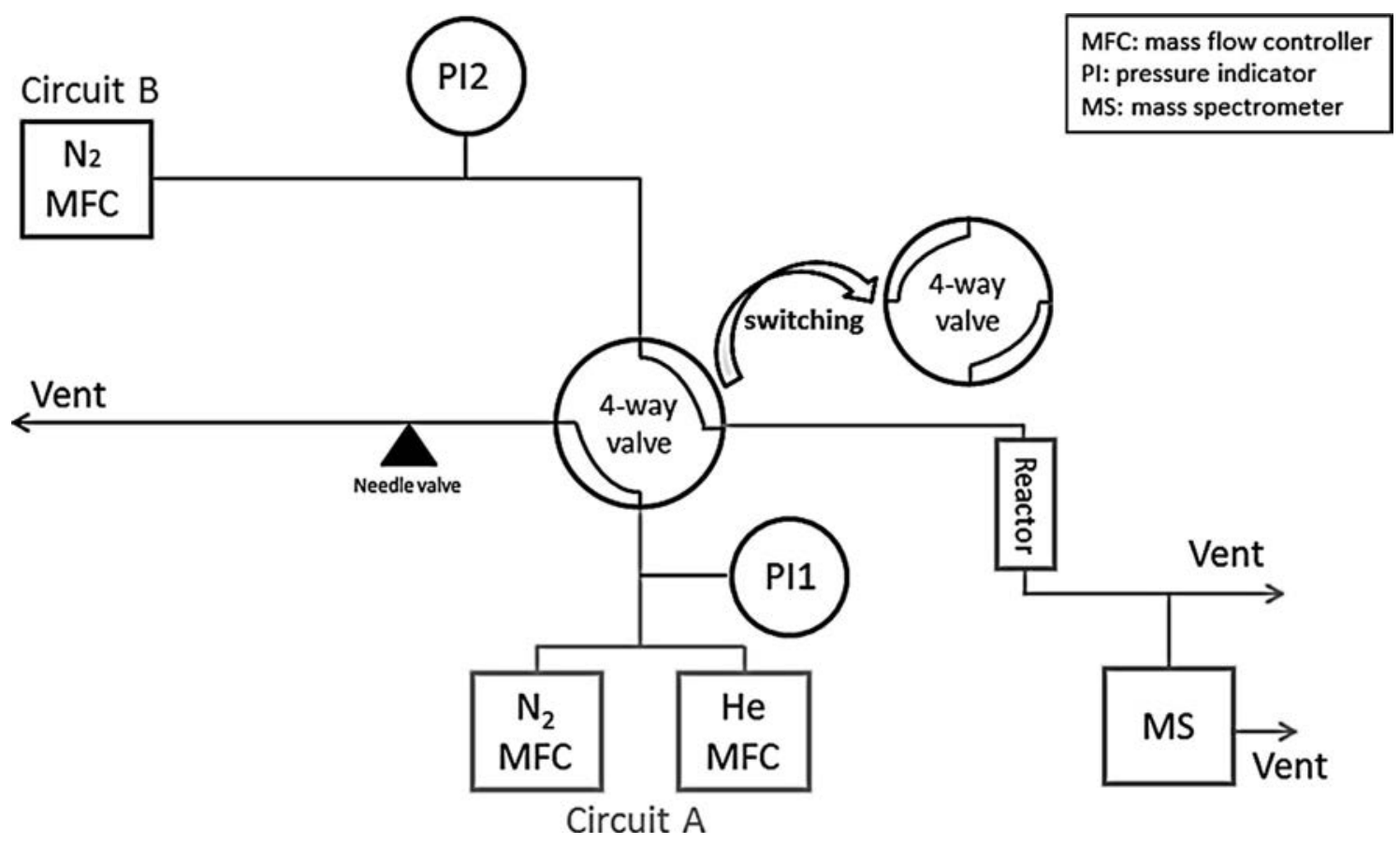

Fig. 2. RTD experimental setup scheme. 


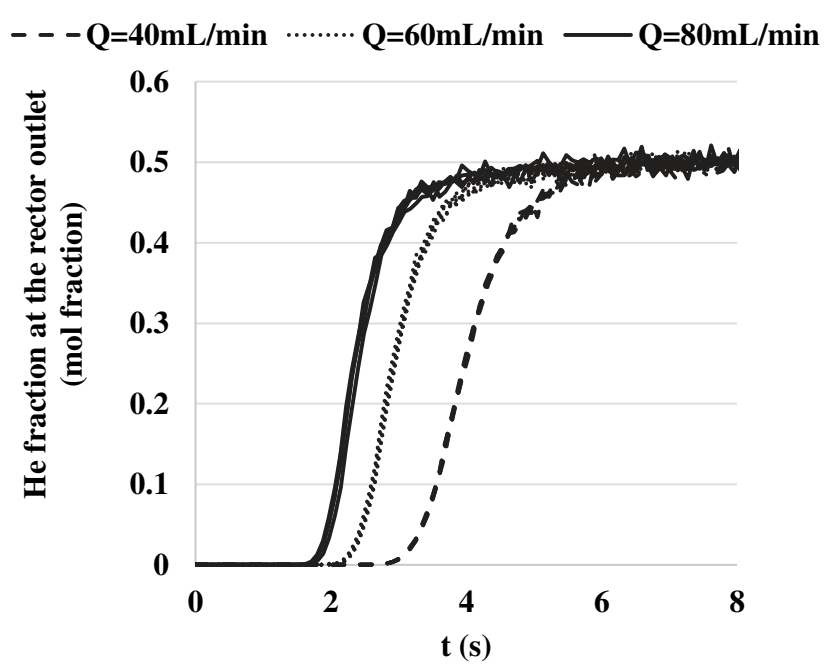

Fig. 3. Evolution of the He molar fraction measured at the reactor outlet after a step change for 3 flow rates and 5 measurements for each flow rate. Reactor with $\mathrm{L}=8.8 \mathrm{~cm}, \mathrm{D}_{\mathrm{r}}=5.8 \mathrm{~mm}, \mathrm{~d}_{\mathrm{p}}=2 \times 5 \mathrm{~mm}$ glass cylinders.

Runge-Kutta solver. Grid size and time step have been refined to ensure grid and time step independence.

For each reactor configuration and condition, the model is used to fit the two unknown parameters: $D_{a x}$, the axial dispersion coefficient, and $\varepsilon$, the void fraction. They have been fitted so that the simulation output matches the experimental "reactor" outlet temporal profiles when inputting the blank temporal profiles (Fig. 4).

More precisely, the value $\varepsilon$ has been first estimated at the lowest flow rate (the longest residence time), to ensure the highest accuracy and this value has been used for the other flow rates of this particular reactor. The value of $D_{a x}$ is estimated by minimizing the relative square error (RSE) between experimental and numerical output (Eq. (4)).

$\sum_{i} \frac{\left(y_{i}^{\text {mod }}-y_{i}^{\exp }\right)^{2}}{y_{i}^{\exp 2}}$

The summation in Eq. (3) is carried out for the helium mol fraction between $0.05 \%$ and $25 \%$. The $0.05 \%$ threshold aims at reducing

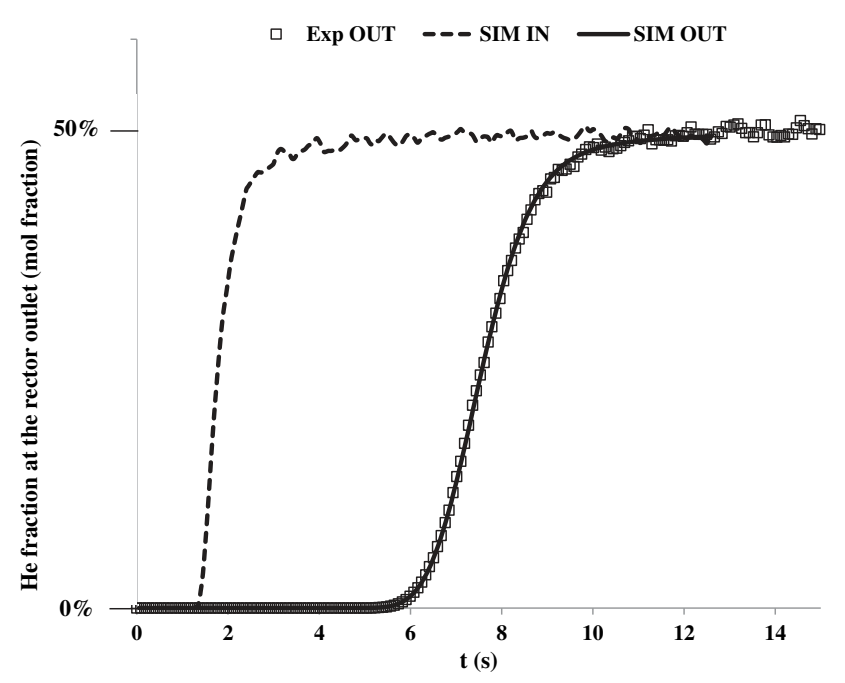

Fig. 4. Model fit of a RTD concentration response curve at the outlet using the axial dispersion model. Exp: experimental, SIM: Simulation. the impact of the "background MS noise" at very low concentration. The upper limit at $50 \%$ of the concentration change is used to reduce the noise observed at high concentration (see signal's tail in Fig. 3). We have defined (arbitrarily) the uncertainty range of $D_{a x}$ as the upper and lower values of $D_{a x}$ such that the value of the maximum relative error on any single point is lower than $20 \%$ (Eq. (5)):

$\max _{i}\left(\frac{y_{i}^{\text {mod }}-y_{i}^{\text {exp }}}{y_{i}^{\exp }}\right) \leq 20 \%$

\subsection{Operating conditions}

The following conditions have been used to measure the dispersion in gas-solid millipacked bed reactors:

- The reactor diameters varied from 2.0 to $7.75 \mathrm{~mm}$ and the lengths from 8.7 to $25 \mathrm{~cm}$;

- The gas flow rates varied from 40 to $120 \mathrm{~mL} / \mathrm{min}$ at $28^{\circ} \mathrm{C}$. The corresponding inlet superficial velocities are from 0.014 to $0.21 \mathrm{~m} / \mathrm{s}$;

- Glass or ceramic non-porous catalyst pellets with cylindrical and spherical shape (1-7 mm) were used to pack the reactors;

- Inert powders (silicon carbide and commercial ZirBlast/ MicroBlast ${ }^{\circledR}$ ceramic powders (Saint Gobain ZirPro) of different sizes were added in some cases to fill the porosity of the beds.

The particle size distributions of the porosity fillers were determined by laser diffraction (Malvern Mastersizer 3000).

A thin layer of glass wool has been used at the top and bottom of the reactor to keep the particles in position inside the reactor.

\subsection{Reactive tests: Unit description and experimental conditions}

The reactive tests have been conducted in a high-throughput catalytic testing setup (Avantium Flowrence ${ }^{\mathrm{TM}}$ ) equipped with 8 parallel fixed bed reactors with internal diameters between 2 and $4 \mathrm{~mm}$ and a catalyst capacity of around $100 \mathrm{mg}$ per reactor.

Two glass chip splitters, one for liquid and one for gas flow, ensure an equal flow distribution over the 8 reactors. Gas and liquid flows are mixed at the reactor inlet. At the reactor outlet, the streams are diluted with nitrogen and sent to a back-pressure regulator that regulates the same inlet pressure for all the reactors. The products composition is measured by on-line gas phase chromatography (one reactor at a time). The effluent is simultaneously analyzed on two channels. The first channel, with argon as carrier gas, has two columns with a Thermal Conductivity Detector. This channel allows the detection of helium, hydrogen, and nitrogen. On the other channel, a Flame Ionization Detector is used for the detection of hydrocarbons. This channel uses hydrogen as a carrier gas and contains a DB1 column, for the separation of light hydrocarbons.

Helium has been introduced in the reactors as an internal standard in order to calculate the outlet flow-rates. Conversions have been calculated as $X\left(\%_{\text {mol }}\right)=100 *\left(1-F_{\text {out }} / F_{\text {in }}\right)$, where $X$ is the conversion and $F$ is the molar flow rate of the reactant. The outlet molar flow rate is calculated using the total outlet flow-rates obtained from the helium conservation balance.

Tests have been performed on two reactions with different thermicity:

- $n$-heptane (nC7) reforming, almost a-thermic $\left(\Delta H^{\circ} \approx 0 \mathrm{~kJ} / \mathrm{mol}\right.$ );

- methylcyclohexane $(\mathrm{MCH})$ dehydrogenation, highly endother$\operatorname{mic}\left(\Delta H^{\circ}=204.8 \mathrm{~kJ} / \mathrm{mol}\right.$ (Kariya et al., 2003). 
Both reactions involve the transformation of a molecule containing 7 carbon atoms over a naphtha reforming catalyst. Pt $/ \gamma-$ $\mathrm{Al}_{2} \mathrm{O}_{3}-\mathrm{Cl}$ extrudates have been used for both reactions, while Pt$\mathrm{Sn} / \gamma-\mathrm{Al}_{2} \mathrm{O}_{3}-\mathrm{Cl}$ spherical beads have been used as a catalyst only for $n$-heptane reforming. The extrudates have a diameter of $1.4 \mathrm{~mm}$ and a length of $5 \pm 2 \mathrm{~mm}$. The beads have a mean diameter of $1.8 \mathrm{~mm}$. The catalysts have been reduced in situ for two hours at $500{ }^{\circ} \mathrm{C}$ under $\mathrm{H}_{2}$ flow before exposition to the gaseous feedstock.

The main reactions for $n$-heptane reforming and for methylcyclohexane dehydrogenation are given in Tables 1 and 2 . The number of moles increases and consequently the volume flow increases in both cases.

$\mathrm{SiC}$ with a Sauter mean diameter $\left(d_{32}\right)$ of 185 and $456 \mu \mathrm{m}$ and ZirBlast $^{\circledR}$ with a Sauter mean diameter $\left(d_{32}\right)$ of 216 and $333 \mu \mathrm{m}$ were used as porosity fillers. Additionally, we tested fine SiC and MicroBlast ${ }^{\circledR}$ powders $(60 \mu \mathrm{m})$. We will refer to the MicroBlast ${ }^{\circledR}$ powder as ZirBlast ${ }^{\circledR}$ for convenience, as it is the same material. The powder addition caused a pressure drop in the reactors of maximum 250 mbar. Considering the reactions in exam, we consider this not affecting the results.

Experiments over the extrudates or beads and the equivalent packing with porosity filler were always run in parallel. This allowed to minimize the effect of catalyst activation or deactivation on the results, as the difference in conversion between two packings is analyzed, which was always less than $6 \%$.

The experimental conditions for the two reactions are presented in Table 3 and Table 4.

In order to explore external mass transfer limitations, we have chosen conditions in which the reaction kinetics is fast, mostly high temperature.

\section{Results and discussion}

\subsection{Plug flow behavior}

The analysis of the temporal concentration profiles of the RTD measurements has confirmed that the axial dispersion model is adequate for the description of the hydrodynamics of millipacked beds with small values of $\delta$ (Fig. 4). The experimental data collected using different reactors and particles, under different flow rates, spanning a range of $\delta$ from 1.1 to 7.75 , are in line with the literature already published for larger packed beds (Fig. 5).

In Fig. 5, Delgado included a theoretical curve derived by assuming additive contribution of two asymptotic dispersions: at very low velocity, the dispersion is equal to the effective diffusion (molecular diffusion divided by the tortuosity of the packed bed), while at high velocity each particle acts as a perfect mixer so that $B o(\infty)=2$ (McHenry and Wilhelm, 1957). This leads to Eqs. (6) and (7):
Table 2

Main reaction for methylcyclohexane dehydrogenation.

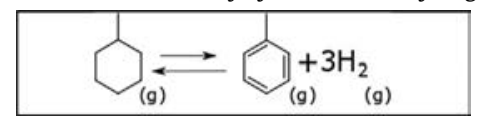

Table 3

Ranges of operating conditions explored in $\mathrm{nC7}$ reforming.

\begin{tabular}{lll}
\hline Condition & Range & Unit \\
\hline Reactor diameter & $2 ; 3 ; 4$ & $\mathrm{~mm}$ \\
Temperature & $450-515$ & ${ }^{\circ} \mathrm{C}$ \\
Total pressure & 1.1 & $\mathrm{MPa}$ \\
$\mathrm{H}_{2} / \mathrm{nC7}$ & 10 & $\mathrm{~mol} / \mathrm{mol}$ \\
WHSV & $5-15$ & $\mathrm{~g}_{\mathrm{nc}} /\left(\mathrm{h}^{*} \mathrm{~g}_{\text {cat }}\right)$ \\
Conversion & $45-90$ & $\%$ \\
\hline
\end{tabular}

Table 4

Ranges of operating conditions explored in $\mathrm{MCH}$ dehydrogenation.

\begin{tabular}{lll}
\hline Condition & Range & Unit \\
\hline Reactor diameter & $2 ; 4$ & $\mathrm{~mm}$ \\
Temperature & $330-350$ & ${ }^{\circ} \mathrm{C}$ \\
Total pressure & 1.1 & $\mathrm{MPa}$ \\
$\mathrm{H}_{2} / \mathrm{MCH}$ & 5 & $\mathrm{~mol} / \mathrm{mol}$ \\
$\mathrm{N}_{2} / \mathrm{H}_{2}$ & 1 & $\mathrm{~mol} / \mathrm{mol}$ \\
WHSV & $5-17.5$ & $\mathrm{~g}$ \\
Conversion & $40-80$ & $\%$ \\
\hline
\end{tabular}

$D_{a x}=\frac{D_{m}}{\tau}+\frac{u * d_{p}}{\varepsilon * 2}$

$\frac{1}{B o}=\frac{1}{\tau} \frac{1}{P e_{m}}+\frac{1}{2}$

At low $P e_{m}\left(\frac{u_{i} * d_{p}}{D_{m}}\right)$ number values, the dispersion is mostly due to diffusion corrected by the bed tortuosity. In all the experimental literature, measurements of Bo numbers overestimate this asymptotic behavior for intermediate values of $P e_{m}\left(P e_{m} \sim 1-10\right)$. Our measurements are in both the molecular diffusion-controlled zone and in the overestimation zone. We measured high values of Bo number $(B o>3)$ for reactors with low values of $\delta$. This is in line with the values reported, for higher gas velocities, by Šolcova and Schneider (Šolcova and Schneider, 2004), Scott et al. (Scott et al., 1974) and Hsiang and Haynes (Hsiang and Haynes, 1977).

Some authors have modified the Eq. (7) in order to predict their experimental data, in particular for the overestimation zone (i.e. Edwards and Richardson (Edwards and Richardson, 1968), Scott et al. (Scott et al., 1974) or Šolcova and Schneider (Šolcova and Schneider, 2004).

Table 1

Main reactions involved in n-heptane reforming.

$\underbrace{\longrightarrow}_{(g)}$

\subsection{Effect of the bed length}

In the explored experimental domain, the Bo number is independent of the length of the reactor. In Fig. 6, we present a single case, but the same was true for all cases. In our experimental domain, the $P e$ number is proportional to the length. For larger packed beds, Han et al. (Han et al., 1985) found the same result for values of $P e_{m} \leq \sim 700$, but for values of $L / D_{r}>70$.

\subsection{Effect of reactor/particle diameter ratio}

Several RTD measurements have been performed in the same reactor $\left(D_{r}=7.75 \mathrm{~mm}, L=18.2 \mathrm{~cm}\right)$, filled with spherical particles of diameters ranging from 1 to $7 \mathrm{~mm}$. The values of $P e$ number and 


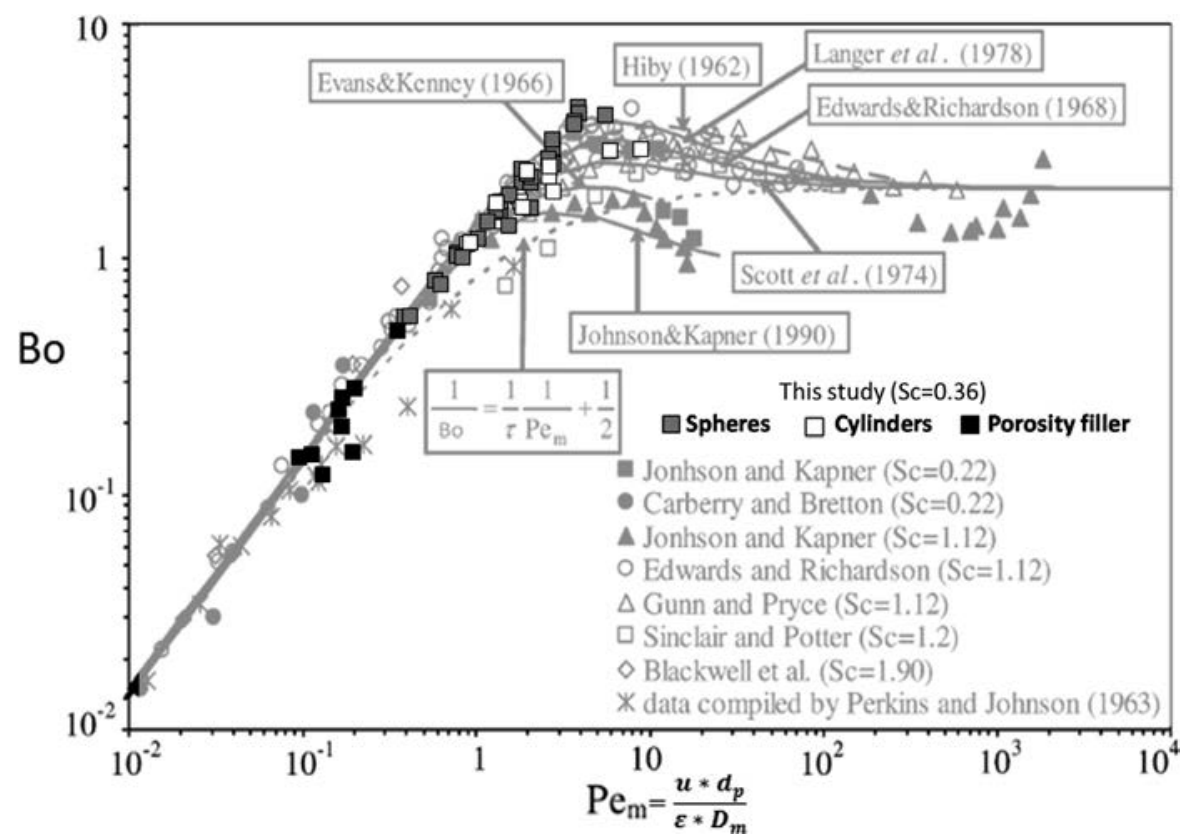

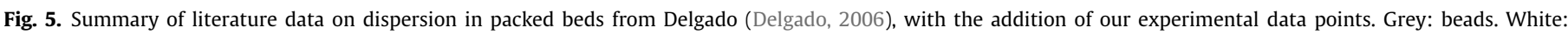
cylinders. Black: beads or cylinders + porosity filler.

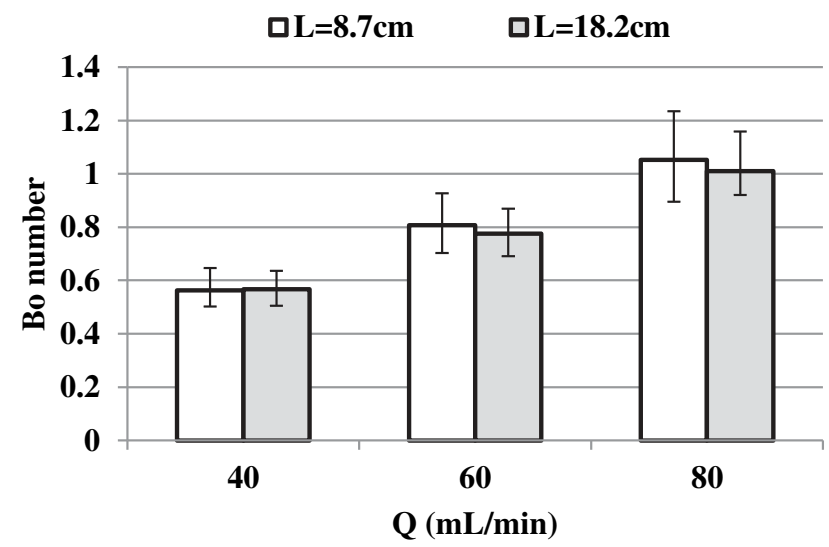

Fig. 6. Variation of Bo number for reactors with $D_{r}=7.75 \mathrm{~mm}$ and $d_{p}=1 \mathrm{~mm}$ beads, all other parameters identical. $\varepsilon$ are shown in Fig. 7 for two values of the superficial gas velocity: $0.014 \mathrm{~m} / \mathrm{s}$ and $0.028 \mathrm{~m} / \mathrm{s}$. The value of the Pe number is increased, but not doubled when doubling the gas velocity.

The Pe number evolution as a function de $\delta$ is complex and not monotonous for $\delta<3$. At both gas velocities, the Pe number presents a minimum for $\delta=1.55$, corresponding to the reactor that has the largest void fraction. In that reactor, the beads arrange in a vertical plane leaving large empty spaces on their side (Fig. 1). This structure is prone to bypasses, which explains the drop in Pe number. Another minimum is present at $\delta=2.58$, but only for the highest velocity $(u=0.028 \mathrm{~m} / \mathrm{s})$. At that $\delta$ value, the beads arrange in a circular manner, all touching the reactor wall and leaving a large empty passage in the reactor center, promoting a severe bypass of the bed and a drop in Pe number compared to structures with not too different $\delta$.

A pronounced increase in Pe occurs when $\delta>3$, especially at the highest velocity. This corresponds to a transition towards beds
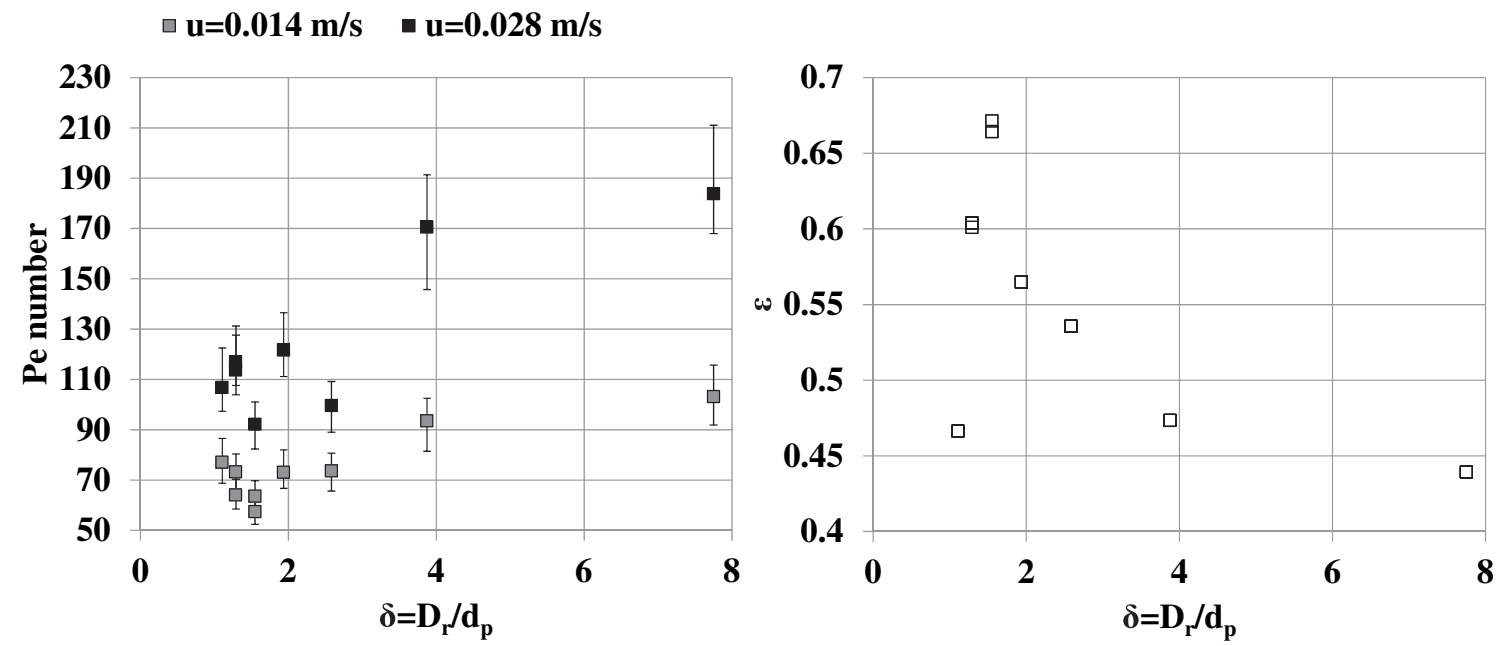

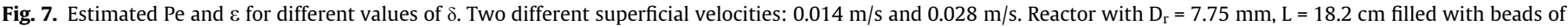
different size. Grey: Pe number values for $\mathrm{u}=0.014 \mathrm{~m} / \mathrm{s}$. Black: Pe number values for $\mathrm{u}=0.028 \mathrm{~m} / \mathrm{s}$. White: $\varepsilon$ values. 
where the packing is less influenced by the presence of the reactor walls and more "uniform". This extends Johnson and Kapner's results (Johnson and Kapner, 1989) towards lower values of $\delta$. Clearly, our experimental sampling of $\delta$ is not sufficient to capture the complete curve. We will report in another paper a more resolved trend obtained using numerical tools.

In the case of cylindrical particles, we define $\psi$ as the ratio between the particle length and the reactor diameter and $\delta$ ' the ratio between the reactor diameter and the particle diameter. Visual observations confirmed that when $\psi$ is larger than 1 the cylindrical particles align along the reactor axis: this leads to structured beds with potential preferential passages between the cylindrical particles and the walls. When $\psi$ is smaller than 1 , the cylinders can position themselves in any direction including horizontally. This promotes a more random and more uniform structure with fewer bypasses. Higher $P e$ numbers have been observed when cylinders are arranged randomly than when they align on each other along the reactor axis (Fig. 8).

\subsection{Effect of superficial gas velocity}

In the case of beads, in a $7.75 \mathrm{~mm}$ diameter reactor, both the axial dispersion coefficient (Fig. 9 left) and Pe number (Fig. 9 right)

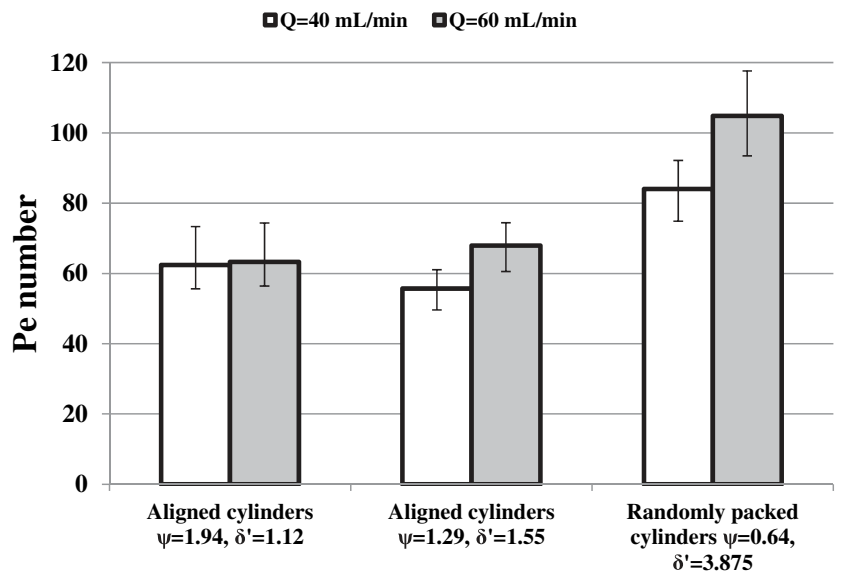

Fig. 8. Pe number for aligned/randomly packed cylinders in a reactor with $D_{r}=7.75$ $\mathrm{mm}, \mathrm{L}=18.2 \mathrm{~cm}$ for two flow rates: 40 and $60 \mathrm{~mL} / \mathrm{min}$. increase with increasing gas velocity. This is expected as our experiments are in the range $0.1<P e_{m}<10$, domain where an increase of velocity corresponds to an increase of the Bo number and, consequently, of the Pe number (see Fig. 5). For higher values of $P e_{m}$ an increase of the velocity could result in a decrease of the Bo number (see Fig. 5 and (Šolcova and Schneider, 2004; Scott et al., 1974; Hsiang and Haynes, 1977).

$D_{a x}$ increases less than linearly with the gas velocity and it converges to $D_{m} / \tau$ at low velocities (see Fig. 5, in the region of $P e_{m}<1$, and Eq. (5)).

The molecular diffusivity of helium in nitrogen is $\sim 7.5 \times 10^{-5}$ $\mathrm{m}^{2} / \mathrm{s}$ at $25{ }^{\circ} \mathrm{C}$. Assuming $\tau=\frac{1}{\sqrt{\varepsilon}}$ (Punčochář and Drahoš, 1993), the tortuosity values for the data shown in Fig. 9 vary between 1.23 and 1.52 and the values of $D_{m} / \tau$ between $\sim 4.9$ and $6.1 \times 10^{-5} \mathrm{~m}^{2} / \mathrm{s}$, which corresponds indeed to the lower end of our $D_{a x}$ values (Fig. 9, left).

This means that at low velocities the Pe number values are mainly driven by the global reactor void fraction, which influences the interstitial velocity (see Fig. $7, u=0.014 \mathrm{~m} / \mathrm{s}$, where the Pe number behavior in inversely proportional to the void fraction). When increasing the velocity, the flow is no longer only governed by the molecular diffusivity, but also by bypasses inside the structures.

\subsection{Effect of inert powder addition as porosity filler}

Experimental data have been acquired with a reactor with $D_{r}=7.75 \mathrm{~mm}, L=18.2 \mathrm{~cm}, u=0.014 \mathrm{~m} / \mathrm{s}$ and packed with glass beads of $5 \mathrm{~mm}$. Silicon carbide (SiC), mean $d_{p}=0.2,0.8 \mathrm{~mm}$ and sieved between $d_{p}=0.3-0.4 \mathrm{~mm}$, and ZirBlast ${ }^{\circledR}(Z B)$, sieved between $d_{p}=0.3-0.4 \mathrm{~mm}$ have been then added to these reactors as porosity fillers. A laser diffraction analysis of the $0.3-0.4 \mathrm{~mm}$ sieved powders gave values of Sauter mean diameter $\left(d_{32}\right)$ of $333 \mu \mathrm{m}$ for the ZirBlast ${ }^{\circledR}$ powder while $456 \mu \mathrm{m}$ for the SiC. The high value observed in case of $\mathrm{SiC}$ is probably due to the irregular shape of the particles. It is in fact possible for long particles with a diameter smaller than $0.4 \mathrm{~mm}$ to pass through the sieve (Fig. 10).

The pressure drop for these reactors has been measured and was found to be insignificant, avoiding any effect on the data analysis.

The porosity fillers are inserted in the beds following a precise protocol:

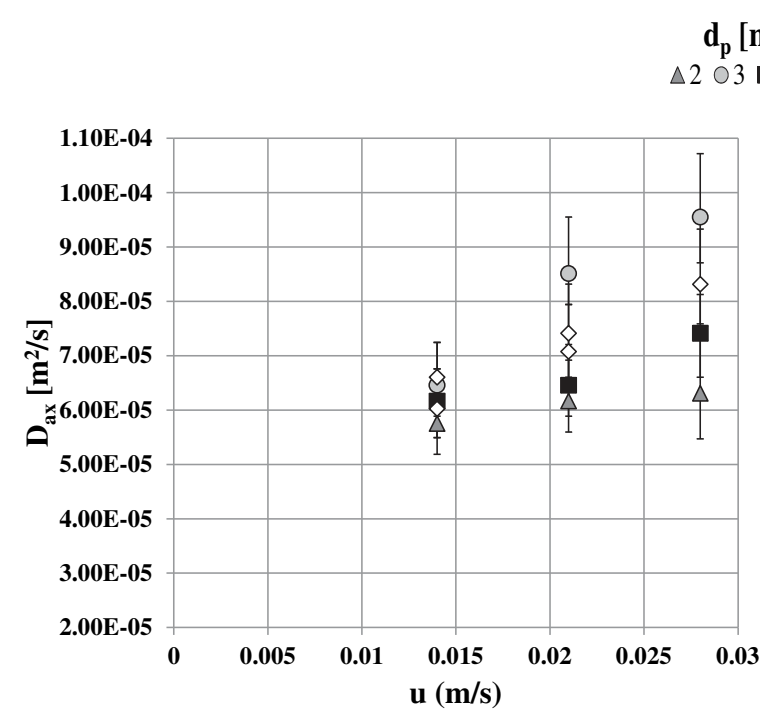

$\mathbf{d}_{\mathrm{p}}[\mathrm{mm}]$

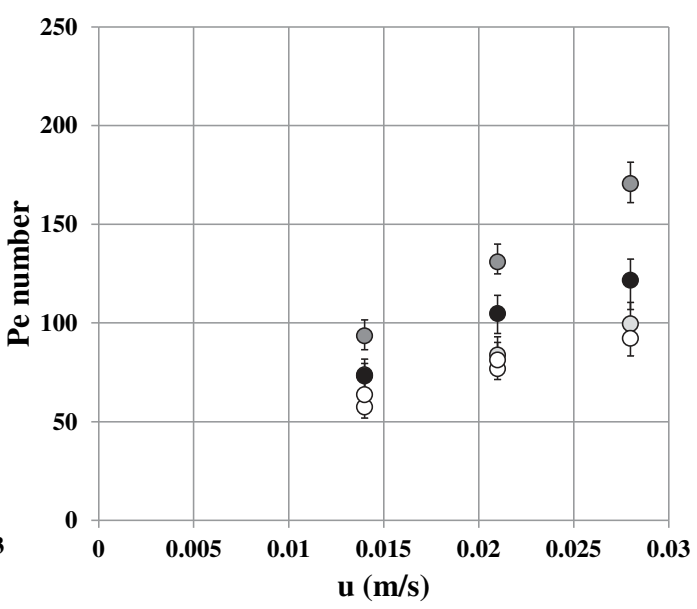

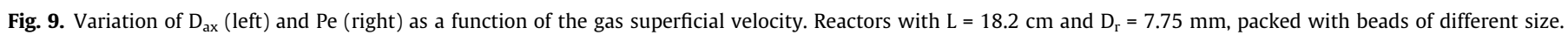



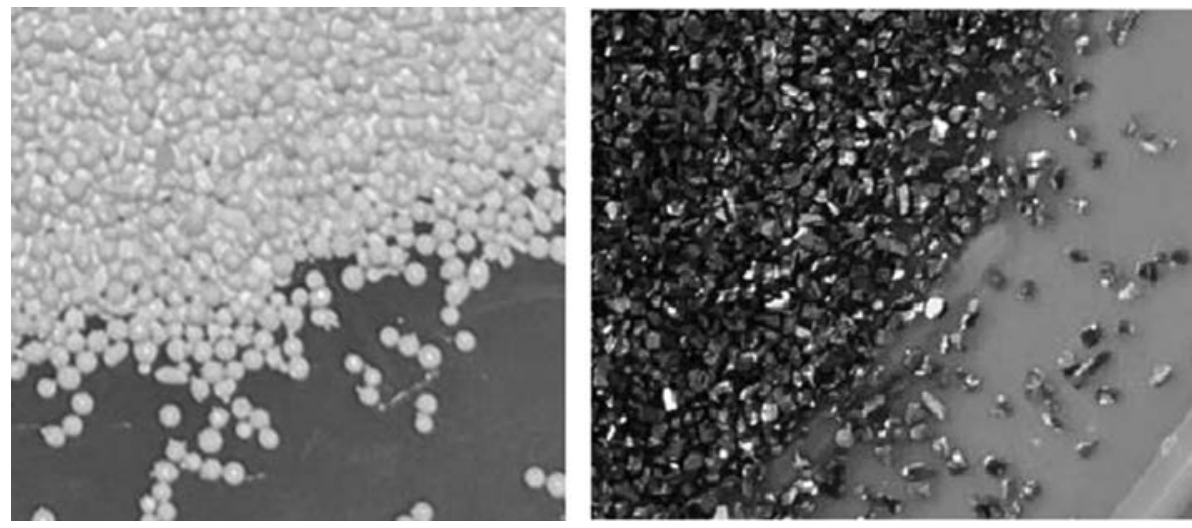

Fig. 10. Photographs of ZirBlast ${ }^{\circledR}$ on the left, SiC on the right.

(1) The reactor is initially packed with the millimetric particles;

(2) Powder is added up to the reactor top;

(3) Gentle tapping during $25 \mathrm{~s}$ is performed. The void created by the rearrangement of the fine powder is filled again with fine powder. This operation is repeated twice.

In one experiment with $\mathrm{SiC} 0.3-0.4 \mathrm{~mm}\left(d_{32}=456 \mu \mathrm{m}\right)$, we repeated the step 3 of the packing protocol three times, instead of two, which reduced the void fraction from 0.39 to 0.37 .

The use of porosity fillers increases significantly the Pe number and this increase is mainly driven by the decrease of the bed void fraction of the reactor (Fig. 11).

Another effect of the porosity filler addition to a bed of millimetric particles is that the size governing the hydrodynamics becomes the porosity filler diameter. The $P e_{m}$ number is thus lower, in the range where the Bo number is proportional to $\mathrm{Pe}_{m}$ (see Fig. 5).

ZirBlast ${ }^{\circledR}$ powder enhances more the Pe number than SiC powder, but this is a direct effect of the void fraction: 0.32 for the bed filled with ZirBlast ${ }^{\circledR}$ while higher for all the beds filled with SiC, even of lower size. This void fraction difference is most likely caused by the shape of the two powders: while the ZirBlast ${ }^{\circledR}$ powder is spherical and flows very well, the $\mathrm{SiC}$ particles have irregular

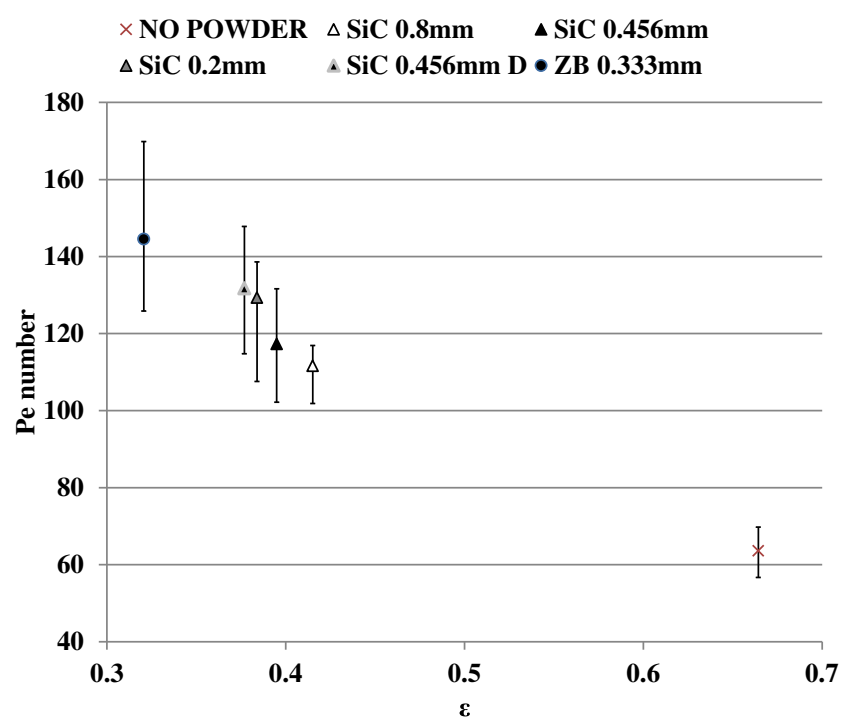

Fig. 11. Pe number against void fraction. Reactor with $D_{r}=7.75 \mathrm{~mm}, L=18.2 \mathrm{~cm}$ and $\mathrm{u}=0.014 \mathrm{~m} / \mathrm{s}$, packed with beads of $5 \mathrm{~mm}$ and different types of porosity fillers. D means "dense packing", obtained repeating 3 times step 3 of the packing protocol. forms and do not fill up the void fraction as the ZirBlast ${ }^{\circledR}$ powder (Fig. 10).

Other experiments, performed with the same reactor $\left(D_{r}=7.75\right.$ $\mathrm{mm})$, but different particles $\left(d_{p}=7 \mathrm{~mm}\right.$ beads and $5 \times 10 \mathrm{~mm}$ cylinders) confirm the positive effect of the powder on the Pe number (Fig. 12).

\subsection{Criterion for axial dispersion limitations}

In this paragraph, we define a criterion for deciding if axial dispersion effects can be neglected for a given reactor, catalyst, and kinetic study. Our derivation is based the Mears-Gierman criterion (Gierman, 1988) combined with a correlation describing our experiments. The Mears-Gierman criterion (Gierman, 1988) gives a minimum $P e$ number allowing to neglect axial dispersion effects without degrading kinetic constant evaluation by more than $10 \%$ (Eq. (8)):

$P e>8 \cdot n \cdot \ln \frac{1}{1-X}$

in which $n$ is the reaction order and $X$ the desired conversion. We can notice that for a first order reaction and a desired conversion of $99 \%$, a Pe number of 40 is enough to neglect the effect of the dispersion on the conversion.

To correlate our data, we adopted the correlation proposed by Šolcova and Schneider (Šolcova and Schneider, 2004) (Eq. (9)):

$\frac{1}{B o}=\frac{\gamma}{P e_{m}}+\frac{\lambda_{0}\left[1-\lambda_{1}\left(d_{p} / D_{r}\right)\left[1+\lambda_{2}(1-\varphi)\right]\right] P e_{m}}{\beta+P e_{m}}$

\section{$\square$ NO POWDER $\square$ POWDER SiC 0.2mm}

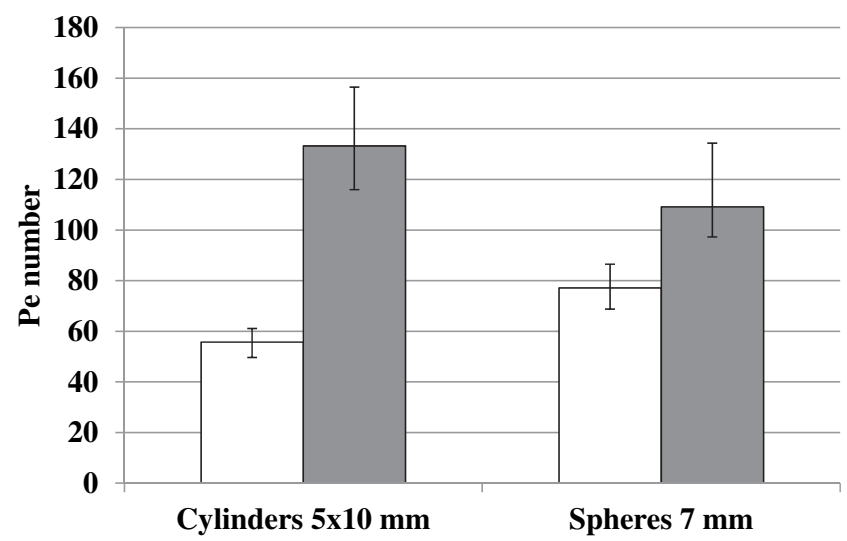

Fig. 12. Porosity filler effect on the Pe number. Reactor with $D_{r}=7.75, L=18.2 \mathrm{~cm}$ and $d_{p}$ indicated in the $\times$ axis. $Q=40 \mathrm{~mL} / \mathrm{min}$. 
in which: $\phi$ represents the particles sphericity $\gamma-\lambda_{0}-\lambda_{1}-\lambda_{2}-\beta$ are constants estimated by fitting the experimental data. The sphericity is defined as the ratio of the surface area of the sphere of the same volume as the particle to the surface area of particle. Thus, for spherical particles $\phi=1$ while for cylindrical particles it is given by:

$\left.\varphi=\frac{18\left(L_{c y l} / D_{c y l}\right)^{2}}{\left(1+2\left(L_{c y l} / D_{c y l}\right)\right)^{3}}\right)^{1 / 3}$

Šolcova and Schneider tested this correlation on both spherical and cylindrical particles and it has been validated for different gas mixtures. However, they studied single pellet string reactors with $1.25<\delta<1.76,1.43<\delta^{\prime}<1.75$ and $0.44<\beta<0.86$, and their correlation does not fit our data points adequately. To fit our experimental points that span a larger range of $\delta, \delta$ ' and $\beta$, we adjusted the $\gamma$ $\lambda_{0}-\lambda_{1}-\lambda_{2}$ coefficients, while keeping the original value of $\beta$.

Minimizing the root mean square error (RMSE) of the relative difference of the experimental data, allowed estimating the values of the coefficients $\gamma-\lambda_{i}$ (denoted as best fit) with a good accuracy. However, the correlation with these values overestimates half of the experimental data points and some by up to $18 \%$. We propose another conservative set of constants $\gamma-\lambda_{i}$ (denoted as conservative), with a lower prediction accuracy, but one that never overestimates the Bo number.

All sets of parameter values and the original ones from Šolcova and Schneider are shown in Table 5.

Experimental data from two reactor configurations are not well predicted by this model:

(1) $\delta=2.58$. This represents a geometry that is characterized by a pronounced preferential passage and a very low Pe value so that the prediction is not accurate. We do not recommend to operate reactors with $\delta$ values close to this value;

(2) $\delta=1.1+\mathrm{SiC} 0.2 \mathrm{~mm}$. This is the only reactor that has a small $\varepsilon$ value without porosity filler (lower than 0.5 while higher than 0.6 for the others). In this reactor, the passages between the walls and the catalyst are quite narrow and the fine powder structure itself is impacted by the presence of the walls. The characteristic dimension for the hydrodynamics is probably not the size of the fine powder.

For all other reactor configurations, we consider the model as an adequate representation of the experimental data.

To summarize, our criterion is based on the following steps:

(1) Use Eq. (9) to estimate the Bo number for the millipacked bed reactor using the set of conservative values from Table 3;

(2) Use Eq. (11) to compute the maximum acceptable conversion. This equation is a direct rewriting of Eq. (8).

$X_{\max }=1-e^{-\frac{L * B o}{8 n d_{p}}}$

\subsection{Reactive tests}

Reactive tests have been performed in order to explore the effect of adding powders as porosity fillers in millipacked bed reac- tors on the external mass transfer. We rank the performances in terms of conversion and assess the effect of the different porosity fillers. We did not wait for stabilization of the catalyst activity because we are interested in the differences between the reactors and not in their absolute values.

The $95 \%$ confidence interval on the differences in conversion is estimated at $\pm 1 \%$. It was estimated through a statistical analysis on the average of the differences of conversion observed in replicated reactors. Both the data on $n$-heptane reforming and methylcyclohexane dehydrogenation were considered in this analysis. The porosity fillers used are the same used in the hydrodynamic study (paragraph §3.1.4), with the addition of $\mathrm{SiC}$ and $\mathrm{ZB}$ powders with Sauter mean diameter $\left(d_{32}\right)$ of 185 and $216 \mu \mathrm{m}$ respectively (obtained through a laser diffraction analysis).

Addition of the porosity fillers lead to a maximum increase of 250 mbar, which is less than $3 \%$ of the total pressure and within the experimental uncertainty.

\subsection{Check of thermal transfer and axial dispersion limitations}

In this section, we check the absence of thermal transfer and axial dispersion limitations.

Mears developed a criterion (Mears, 1971) to predict the presence of radial temperature gradients in large fixed beds in the case where only the wall temperature is known and not the center temperature, which is our case. Radial thermal transfer limitations in a packed bed can be neglected if the condition in Eq. (12) is respected:

$\left.\Delta T_{\text {rad }}=1+\frac{8}{\frac{\alpha_{w} * D_{r}}{\lambda}}\right) *\left|\frac{\Delta H_{0} * r * d_{p}^{2} *(1-\varepsilon)}{32 * \lambda}\right|<\frac{0.05 * R * T^{2}}{E_{a}}$

in which $\Delta T_{\text {rad }}$ is temperature difference between the bed near the wall and the average in the bed, $\alpha_{w}$ is the wall heat transfer coefficient, $\lambda$ is the thermal conductivity of the packed bed, $r$ the volumetric reaction rate, $T$ the absolute temperature, and $E_{a}$ the activation energy of the reaction. This criterion is usually stricter than the one for axial thermal transfer (Mears, 1976). When the thermal Mears radial criterion is fulfilled, axial temperature profiles are also negligible. In practice, the Mears radial criterion is at most indicative as it requires the estimation of two quantities, the wall heat transfer coefficient $\alpha_{w}$ and the thermal conductivity $\lambda$, for which correlations in literature (Yagi and Wakao, 1959; Hanrattry, 1954; De Wasch and Froment, 1972; Chi-Hsiung and Finlayson, 1977; Specchia and Sicardi, 1980; Gunn et al., 1987; Bey and Eigenberger, 2001), give a wide range of estimations. These correlations have been developed for large packed bed reactors and their validity for millipacked beds with low values of $\delta$ is unknown.

According to our calculations, considering the cases at the highest temperature, the criterion is always fulfilled for $n$-heptane reforming. For the methylcyclohexane dehydrogenation, which is highly endothermic, most, but not all correlations used for predicting $\alpha_{w}$ and $\lambda$ allowed to fulfill the criterion.

For this reason, we performed an experimental check on the isothermicity of the methylcyclohexane dehydrogenation by comparing runs with a single bed of catalyst and a reactor, denoted diluted, whose catalyst bed has been split into two parts separated by a bed of highly conductive inert SiC particles. The inert layer will

Table 5

The two set of constants (best fit and conservative fit) for the model in Eq. (9).

\begin{tabular}{|c|c|c|c|c|c|c|}
\hline & $\gamma$ & $\lambda_{0}$ & $\lambda_{1}$ & $\lambda_{2}$ & $\beta$ & RMSE \\
\hline Šolcova and Schneider & 1.142 & 5.311 & 1.169 & 2.296 & 39.76 & \\
\hline Best fit (min RMSE) & 0.763 & 1.786 & 0.629 & 3.708 & 39.76 & 0.07 \\
\hline Conservative fit & 0.934 & 1.605 & 0.639 & 4.420 & 39.76 & 0.17 \\
\hline
\end{tabular}


promote reheating of the fluid at the inlet of the second catalytic bed. In the case of heat transfer limitations, we expect higher conversion in the diluted reactor. The conversion in the diluted reactor was in fact lower.

Eqs. (9) and (11), using the "conservative fit", showed that under our operating conditions dispersion effects can be neglected. We confirmed this conclusion using the analytical solution for a first order reaction in absence of mass transfer limitations. Conversion differences when adding porosity fillers can only be attributed to changes in mass transfer.

\subsection{Porosity filler effect on the conversion: $n$-heptane reforming reaction}

In this part, we will compare the conversions of the reactors packed with and without porosity filler for $n$-heptane reforming reaction. We have measured, for different operating conditions, the difference $\Delta X=X_{\text {filler }, i}-X_{\text {no_filler }}$ where $X_{\text {filler, } i}$ represents the conversion with the porosity filler $i$, while $X_{\text {no_filler }}$ the conversion with- out porosity filler. For each test we consider the average of the different $\Delta X$ calculated in order to assess the effect of the porosity filler and compare it to the $95 \%$ confidence interval. The effect on the conversion is indicated with a "+" if adding the porosity filler increases the conversion compared to the case without porosity filler. When a reactor has a duplicate in a test, the $X$ values retained are the mean of the measured conversions for each particular condition.

\subsection{Catalyst extrudates}

The differences in conversion between the reactors packed with and without porosity filler for some of the operating conditions are shown in Fig. 13 ( 5 tests) while Table 6 summarizes the effects observed for all the results. The addition of porosity fillers had always a beneficial effect on the conversion (see Table 7).

As discussed above, the conversion gains are necessarily due to changes in the external mass transfer. A likely explanation is the decrease of the void fraction and thus the increase of the intersti-

\section{nC7 reforming}
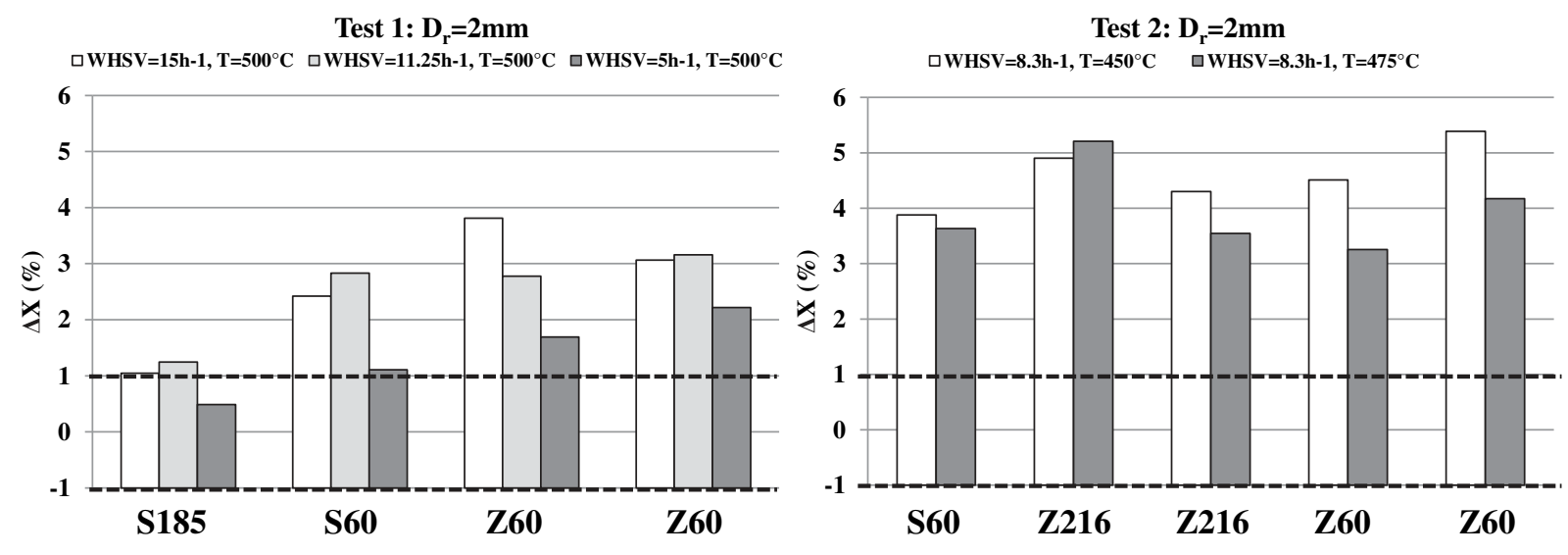

$\square \mathrm{WHSV}=11.25 \mathrm{~h}-1, \mathrm{~T}=470^{\circ} \mathrm{C} \square \mathrm{WHSV}=10 \mathrm{~h}-1, \mathrm{~T}=470^{\circ} \mathrm{C}$

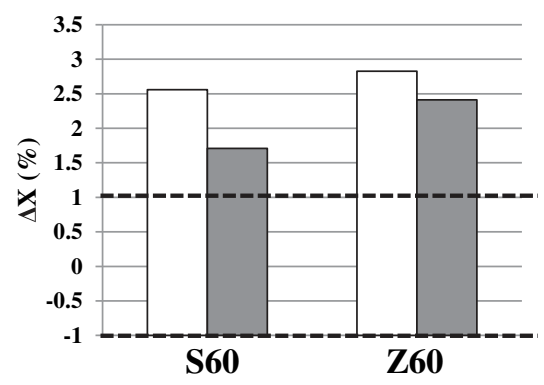

Test 4: $D_{\mathrm{r}}=\mathbf{4 m m}$

$\square \mathrm{WHSV}=7.5 \mathrm{~h}-1, \mathrm{~T}=470^{\circ} \mathrm{C} \quad \square \mathrm{WHSV}=6.6 \mathrm{~h}-1, \mathrm{~T}=470^{\circ} \mathrm{C}$

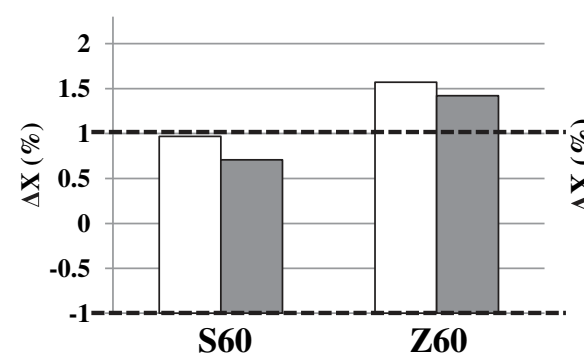

Test 5: $D_{\mathrm{r}}=4 \mathrm{~mm}$

$\square \mathrm{WHSV}=6.6 \mathrm{~h}-1, \mathrm{~T}=470^{\circ} \mathrm{C} \quad \square \mathrm{WHSV}=5.83 \mathrm{~h}-1, \mathrm{~T}=470^{\circ} \mathrm{C}$

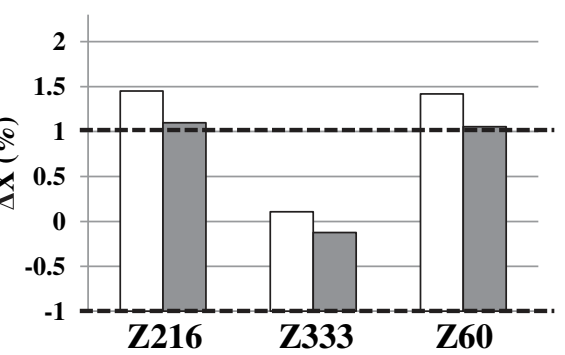

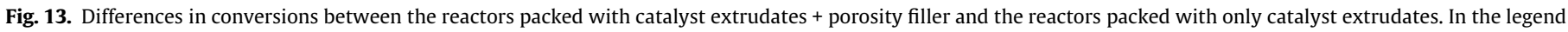
are indicated the type of porosity filler $\left(\mathrm{S}=\mathrm{SiC}, \mathrm{Z}=\mathrm{ZirBlast}^{\circledR}\right)$ and their size in $\mu \mathrm{m}$. The dotted line indicates the confidence interval $( \pm 1.0 \%)$. 
Table 6

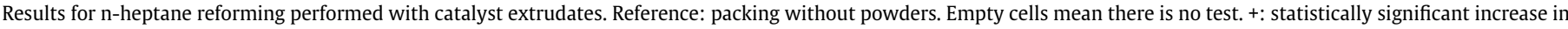
conversion when adding this type of powder.

\begin{tabular}{|c|c|c|c|c|c|c|}
\hline \multirow[t]{2}{*}{$n$-heptane reforming } & & \multicolumn{3}{|c|}{ ZirBlast $^{(\mathbb{R}}$} & \multicolumn{2}{|l|}{$\mathrm{SiC}$} \\
\hline & & $60 \mu \mathrm{m}$ & $216 \mu \mathrm{m}$ & $333 \mu \mathrm{m}$ & $60 \mu \mathrm{m}$ & $185 \mu \mathrm{m}$ \\
\hline Test 1 and $2: D_{r}=2 \mathrm{~mm}$ & WHSV $=5-15 \mathrm{~h}^{-1}$ & + & + & & + & + \\
\hline Test 3: $\mathrm{D}_{\mathrm{r}}=3 \mathrm{~mm}$ & WHSV $=8.8-11.25 \mathrm{~h}^{-1}$ & + & & & + & \\
\hline Test 4 and $5: D_{r}=4 \mathrm{~mm}$ & WHSV $=5.83-6.6 \mathrm{~h}^{-1}$ & + & + & No effect & No effect & \\
\hline
\end{tabular}

Table 7

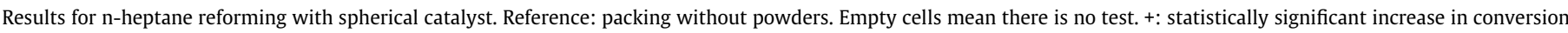
when adding this type of powder.

\begin{tabular}{|c|c|c|c|c|}
\hline \multirow[t]{2}{*}{$n$-heptane reforming } & & \multirow{2}{*}{$\begin{array}{l}\text { ZirBlast }^{\circledR} \\
60 \mu \mathrm{m}\end{array}$} & \multicolumn{2}{|l|}{$\mathrm{SiC}$} \\
\hline & & & $60 \mu \mathrm{m}$ & $456 \mu \mathrm{m}$ \\
\hline $\begin{array}{l}\text { Test 1: } \\
\qquad \begin{array}{l}\mathrm{D}_{\mathrm{r}}=3 \mathrm{~mm} \\
\delta=1.67\end{array}\end{array}$ & WHSV $=8.8-11.25 \mathrm{~h}^{-1}$ & & No effect & + \\
\hline $\begin{array}{l}\text { Test 2: } \\
\qquad \begin{array}{l}\mathrm{D}_{\mathrm{r}}=4 \mathrm{~mm} \\
\delta=2.22\end{array}\end{array}$ & WHSV $=5.4-6.1 \mathrm{~h}^{-1}$ & No effect & No effect & \\
\hline
\end{tabular}

tial velocity, which is known to improve the external mass transfer. Mass transfer coefficient correlations in the literature, for example that of Ranz-Marshall (Ranz and Marshall, 1952), usually relate the external mass transfer coefficient $K_{g s}$ and the fluid velocity with a relationship in the form of Eq. (13):

$K_{g s} \propto a+b * u_{i}^{c}$

where $a, b$ and $c$ are constants. In most correlations, the value of a is such that the $S h$ number equals 2 for $u_{i}=0$, which is the theoretical asymptotic value for a creeping flow around a single sphere. The value of the exponent $c$ is in literature in the range of 0.5-0.6 (Ranz and Marshall, 1952; Wakao and Funazkri, 1978). So, the external mass transfer coefficient increases with increasing interstitial velocity.

The effect of the porosity filler on the conversion is more pronounced for the smaller reactors. The extrudates catalysts have a $1.4 \mathrm{~mm}$ diameter and a $5 \pm 2 \mathrm{~mm}$ length. The extrudates particles are vertically aligned in the reactor of $D_{r}=2 \mathrm{~mm}$, but are randomly oriented in the reactor of $D_{r}=4 \mathrm{~mm}$. The enhancement of mass transfer induced by the porosity filler is thus lower when the reactor structure is random and presents a lesser risk of bypass.

Another trend is that increasing the WHSV tends to increase the gains in conversion when adding porosity fillers. As we operate at constant catalyst mass, increasing the WHSV is equivalent to increasing the gas velocity. When the formalism in Eq. (13) applies, this indicates that the exponent obtained using porosity fillers is higher than the one obtained with millimetric particles only.

\subsection{Catalyst beads}

The effect of the porosity fillers on the conversion with spherical beads is presented in Fig. 14 for some operating conditions. All the results are summarized in Table 5 . The effect of the porosity fillers using beads is less pronounced than for extrudates, not always significantly changing the conversion. The catalyst beads have a mean diameter $d_{p}=1.8 \mathrm{~mm}$, not always perfectly spherical, and they have been tested in reactors with internal diameters of 3 and $4 \mathrm{~mm}$. In the $3 \mathrm{~mm}$ reactor $(\delta=1.67)$, the catalyst beads are positioned in a planar manner with large passages on the side. In the $4 \mathrm{~mm}$ reactor $(\delta=2.22)$, the catalyst beads are arranged irregularly due to the shape and the size distribution of the particles, generating a structure with few bypasses.

The higher effect of the porosity filler in the $3 \mathrm{~mm}$ case is probably due to the presence of large passages between the catalyst particles and the wall. The conversion levels with the spherical beads are consistently higher than with the extrudates. As the cat-
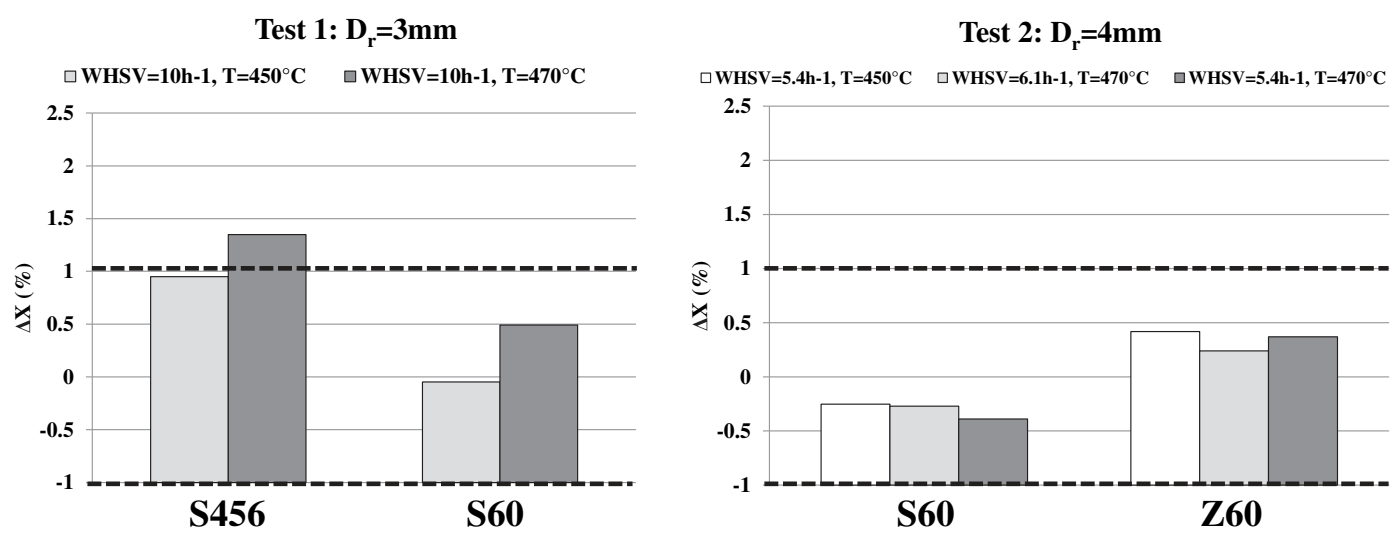

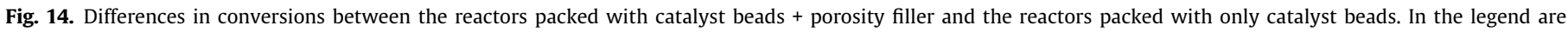
indicated the type of porosity filler $\left(\mathrm{S}=\mathrm{SiC}, \mathrm{Z}=\right.$ ZirBlast $\left.^{\circledR}\right)$ and their size in $\mu \mathrm{m}$. The black dotted line indicates the confidence interval $( \pm 1.0 \%)$. 
alyst formulation is different, it is not possible to distinguish between kinetic or mass transfer effects.

\subsection{Porosity filler size and shape effect}

The effect of the porosity filler type (SiC or ZirBlast ${ }^{\circledR}$ ) was studied by calculating differences in conversion $\Delta X_{p}=X_{Z i r B l a s t, i}-X_{S i C, I}$, where $X_{Z i r B l a s t, i}$ or SiC,i represents the conversion of the packings with the porosity filler ZirBlast ${ }^{\circledR}$ or SiC of size i. In most cases, the use of ZirBlast ${ }^{\circledR}$ results in higher conversions, +0.5 points of conversion on average. The difference for each individual test is not statistically significant, but the effect has been observed repeatedly and it is on average significant (the confidence interval on the average is divided by the square root of the number of repetitions).

This higher performance of ZirBlast ${ }^{\circledR}$ was also observed on the Pe number and explained by a better filling of the porosity. We propose that the slight mass transfer enhancement also results from a reduced void fraction, due to a higher interstitial velocity and higher mass transfer when filling the porosity with ZirBlast ${ }^{\circledR}$ than with SiC. ZirBlast ${ }^{\circledR}$ powder, that is spherical, flows better and fills better the void spaces than $\mathrm{SiC}$ powder that is angular.

The effect of powder size is not statistically significant in our experiments. The conclusion is that the powder size shows no or very little effect. A detailed look at Tables 4 and 5 combined with our remarks on bypasses in the reactors hints that a large porosity filler increase the performance in presence of large passages, whereas a fine porosity filler is more performant in structures with smaller passages. The optimal powder size depends on the bypass size.

\subsection{Porosity filler effect on the conversion: Methylcyclohexane dehydrogenation reaction}

Extrudates catalyst particles have also been used for methylcyclohexane dehydrogenation in reactors of internal diameters of 2 and $4 \mathrm{~mm}$.
For methylcyclohexane dehydrogenation, the differences in conversion between the reactors packed with and without porosity filler are summarized in Table 8 (2 tests) and in Fig. 15 for some operating conditions. The addition of porosity fillers has always a beneficial effect on the conversion with a more pronounced effect in the $2 \mathrm{~mm}$ reactor.

Compared to $n$-heptane reforming, we observe the same trends: a larger mass transfer enhancement when increasing the velocity (WHSV) and no clear effect of powder size. The SiC/ZirBlast ${ }^{\circledR}$ difference is, however, not observed.

\section{Conclusions}

Gas-solid RTD experiments and reactive tests on $n$-heptane reforming and methylcyclohexane dehydrogenation have been performed in millipacked bed reactors with low values of the reactor/particle diameter ratio $(1<\delta<8)$ in order to assess the hydrodynamic and mass transfer characteristics of these reactors.

The axial dispersion model was found adequate to model the RTD curves of these reactors. The dispersion values were found in the range of traditional packed bed reactors for the area explored $\left(0.1<P e_{m}<10\right)$ except that the linear relationship between $\mathrm{Pe}_{m}$ and $\mathrm{Bo}$ numbers extends until $\mathrm{Bo}>3$. For both cylinders and beads, the reduction of the particle size has a significant impact on the packing structure and the values of the $P e$ number. For beads, the experimental resolution on $\delta$ was not sufficient to capture the complex dependency of $P e$ number with $\delta$, which also depends on the gas velocity. The values of the Pe number present minima when the structure is prone to create bypasses. It increases when $\delta>3$. A CFD study is ongoing to explore the range $1<\delta<3$. For cylinders, the Pe number is lower when the cylinders are constrained to align vertically compared to when they can lay horizontally and form a random structure $(\psi>1)$.

Adding fine inert powder to fill the porosity increases the $\mathrm{Pe}$ number through a reduction of the void fraction and the related velocity increase. Additionally, as the hydrodynamic characteristic diameter is reduced, the $P e_{m}$ number is low enough for the Bo

Table 8

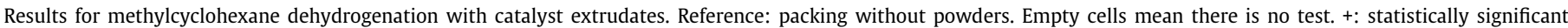
increase in conversion when adding this type of powder.

\begin{tabular}{|c|c|c|c|c|c|c|c|}
\hline \multicolumn{2}{|c|}{ Methylcyclohexane dehydrogenation } & \multicolumn{3}{|c|}{ ZirBlast $^{\circledR}$} & \multicolumn{3}{|l|}{$\mathrm{SiC}$} \\
\hline & & $60 \mu \mathrm{m}$ & $216 \mu \mathrm{m}$ & $333 \mu \mathrm{m}$ & $60 \mu \mathrm{m}$ & $185 \mu \mathrm{m}$ & $456 \mu \mathrm{m}$ \\
\hline Test 1: $\mathrm{D}_{\mathrm{r}}=2 \mathrm{~mm}$ & WHSV $=7.5-17.5 \mathrm{~h}^{-1}$ & + & + & & + & + & \\
\hline Test 2: $\mathrm{D}_{\mathrm{r}}=4 \mathrm{~mm}$ & WHSV $=5-6.67 \mathrm{~h}^{-1}$ & + & + & + & + & + & + \\
\hline
\end{tabular}
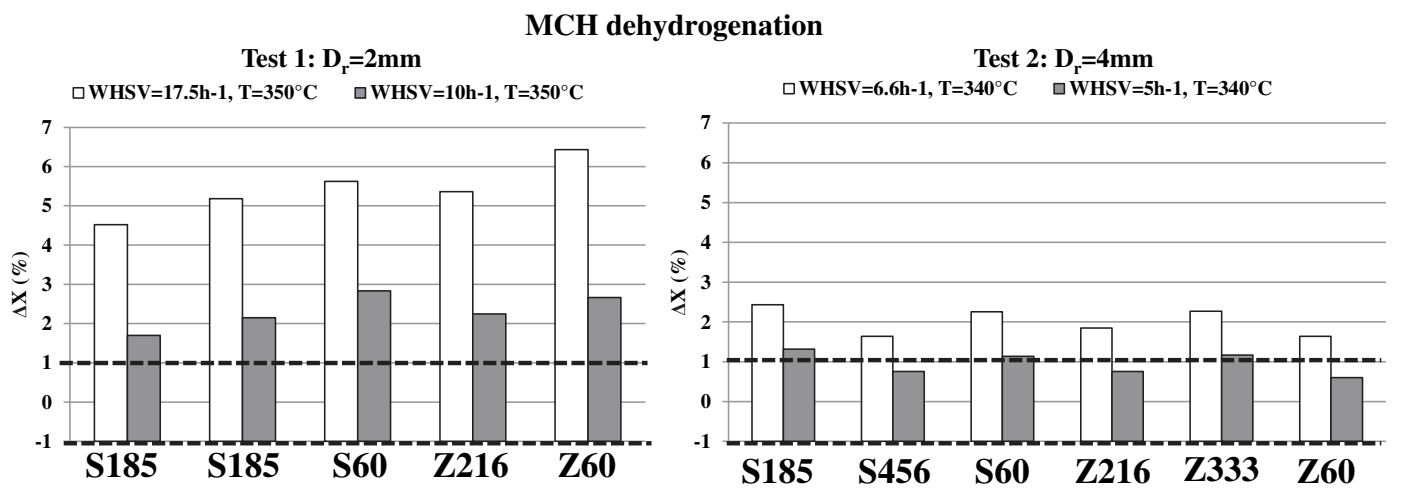

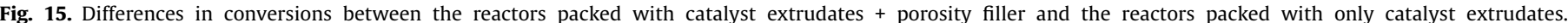

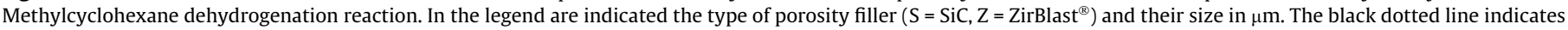
the confidence interval $( \pm 1.0 \%)$. 
number to remain proportional to $P e_{m}$ number, even at high velocities. The best porosity filler is the one that fills up best the entire void fraction, especially near the contact points: it is preferably round (better flowability) and as small as possible (better access to stranded zones near particles contact points). Overall millipacked bed reactors have shown, in the majority of cases, quite good hydrodynamics for most applications. A criterion to calculate, in a conservative manner, the maximum conversion that can be achieved neglecting the dispersion effects is proposed. Some cases where this model is not predictive have been identified (at least 2 identified here: $\delta=2.58$ and for $\delta=1.1+$ porosity filler).

Reactive tests showed that using porosity filler can increase the external gas-solid mass transfer, which we think is due to the lower void fraction that leads to a higher interstitial gas velocity. Spherical powders are again recommended as they yield lower void fractions for a given grain size. The effect of the powder diameter is below our experimental capabilities, but we do not recommend too fine grain sizes, but the one small enough to correctly fill the bed porosity.

At last, our work also indicates that the data reproducibility for HTE systems may be limiting types of kinetic studies requiring high accuracy and investigating very small differences, except at the cost of repeating the tests several times. The variability seems to come from the flow distribution control, that is quite difficult for small flow-rates, and the very small amounts of catalyst tested that raise questions about the catalyst sample uniformity.

\section{CRediT authorship contribution statement}

Vittorio Petrazzuoli: Investigation, Data curation. Matthieu Rolland: Supervision, Project administration, Writing - review \& editing. Adrien Mekki-Berrada: Supervision, Writing - review \& editing. Olivier Said-Aizpuru: Investigation, Data curation. Yves Schuurman: Supervision, Writing - review \& editing.

\section{Declaration of Competing Interest}

The authors declare that they have no known competing financial interests or personal relationships that could have appeared to influence the work reported in this paper.

\section{References}

Bey, O., Eigenberger, G., 2001. Gas flow and heat transfer through catalyst filled tubes. Int. J. Therm. Sci. 40, 152-164.

Chi-Hsiung, Li, Finlayson, B.A., 1977. Heat transfer in packed beds - a reevaluation. Chem. Eng. Sci. 32, 1055-1066.

Chu, C.F., Ng, K.M., 1989. Flow in packed tubes with a small tube to particle diameter ratio. AIChE J. 35 (1).

De Wasch, A.P., Froment, G.F., 1972. Heat transfer in packed beds. Chem. Eng. Sci. 27, 567-576.

Delgado J.M.P.Q., 2006. A critical review of dispersion in packed beds, Heat Mass Transfer, 42(4), Pag. 279-310. DOI: 10.1007/s00231-005-0019-0.

Edwards, M.F., Richardson, J.F., 1968. Gas dispersion in packed beds. Chem. Eng. Sci. 23, 109-123.

Gierman, H., 1988. Design of laboratory hydrotreating reactors. Scaling down of trickle-flow reactors. Appl. Catal. 43 (2), 277-286. https://doi.org/10.1016/ S0166-9834(00)82732-3.
Gunn, D.J., Ahmad, M.M., Sabri, M.N., 1987. Radial heat transfer to fixed beds of particles. Chem. Eng. Sci. 42 (9), 2163-2171.

Han, N.W., Bhakta, J., Carbonell, R.G., 1985. Longitudinal and lateral dispersion in packed beds: effect of column length and particle size distribution. AIChE J. 31 (2), 277-288.

Hanrattry, T.J., 1954. Nature of wall heat transfer coefficient in packed beds. Chem. Eng. Sci. 5, 209-214.

Hsiang, T.C.S., Haynes Jr, H.W., 1977. Axial dispersion in small diameter beds of large, spherical particles. Chem. Eng. Sci. 32, 678-681.

Johnson, G.W., Kapner, R.S., 1989. The dependence of axial dispersion on nonuniform flows in beds of uniform packing. Chem. Eng. Sci. 45 (11), 3329-3339.

Kariya, N., Fukuoka, A., Utagawa, T., Sakuramoto, M., Goto, Y. Ichikawa, M., 2003. Efficient hydrogen production using cyclohexane and decalin by pulse-spray mode reactor with Pt catalysts. Appl. Catal. A 247 (2), 247-259. https://doi.org/ 10.1016/S0926-860X(03)00104-2.

Klyushina, A., Pacultová, K., Obalová, L., 2015. Advantage of the single pellet string reactor for testing real-size industrial pellets of potassium-doped CoMnAl catalyst for the decomposition of N2O. Reac Kinet Mech Cat 115 (2), 651-662. https://doi.org/10.1007/s11144-015-0871-y.

Márquez, N., Castaño, P., Makkee, M., Moulijn, J.A., Kreutzer, M.T., 2008. Dispersion and holdup in multiphase packed bed microreactors. Chem. Eng. Technol. 31 (8), 1130-1139. https://doi.org/10.1002/ceat.200800198.

McGeary, R.K., 1961. Mechanical packing of spherical particles. J. Am. Ceram. Soc. 44 (10), 513-522.

McHenry, K.W., Wilhelm, R.H., 1957. Axial mixing of binary gas mixtures flowing in a random bed of spheres. AIChE J. 3 (1), 83-91. https://doi.org/10.1002/ aic.690030115.

Mears, D.E., 1971. Diagnostic criteria for heat transport limitations in fixed bed reactors. J. Catal. 20, 127-131.

Mears, D.E., 1976. On criteria for axial dispersion in nonisothermal packed-bed catalytic reactors. Ind. Eng. Chem. Fundam. 15 (1), 20-23.

Moonen R., Alles J., Ras E.-j., Harvey C., Moulijn J.A., 2017. Performance testing of hydrodesulfurization catalysts using a Single-Pellet-String Reactor, Chem. Eng. Technol., 40(11) 2025-2034. DOI: 10.1002/ceat.201700098.

Moulijn J.A., Pérez-Raḿırez J., Berger R.J., Hamminga G., Mul G., Kapteijn F., 2003. High-throughput experimentation in catalyst testing and in kinetic studies for heterogeneous catalysis. Catal. Today 81(3) 457-471. DOI: 10.1016/S0920-5861 (03)00145-7.

Mueller, G.E., 1992. Radial void fraction distributions in randomly packed fixed beds of uniformly sized spheres in cylindrical containers. Powder Technol 72 (3), 269-275. https://doi.org/10.1016/0032-5910(92)80045-X.

Punčochář, M., Drahoš, J., 1993. The tortuosity concept in fixed and fluidized bed. Chem. Eng. Sci. 48 (11), 2173-2175. https://doi.org/10.1016/0009-2509(93) 80092-5.

Ranz, W.E., Marshall, W.R., 1952. Evaporation from drops. Chem. Eng. Prog. 48 (3), $141-146$.

Rolland, M., Fonte, C.P., 2015. Incertitude induced by testing a small number of catalytic pellets in fixed beds. Chem. Eng. Sci. 138, 698-705. https://doi.org/ 10.1016/j.ces.2015.09.008.

Saint Gobain ZirPro Website https://www.zirpro.com/.

Scott, D.S., Lee, W., Papa, J., 1974. The measurement of transport coefficients in gassolid heterogeneous reactions. Chem. Eng. Sci. 29, 2155-2167.

Sie, S.T., 1996. Miniaturization of hydroprocessing catalyst testing systems: Theory and practice. AIChE J. 42 (12), 3498-3507.

Šolcova, O., Schneider, P., 2004. Axial dispersion in single pellet-string columns with non-porous packing. Chem. Eng. Sci. 59 (6), 1301-1307. https://doi.org/ 10.1016/j.ces.2004.01.011

Specchia, V., Sicardi, S., 1980. Heat transfer in packed bed reactors with one phase flow. Chem. Eng. Commun. 4 (1-3), 361-380.

Templis, C.C., Papayannakos, N.G., 2017. Liquid-to-particle mass transfer in a structured-bed minireactor. Chem. Eng. Technol. 40 (2), 385-394. https://doi. org/10.1002/ceat.201500733.

Wakao, N., Funazkri, T., 1978. Effect of fluid dispersion coefficients on particle-tofluid mass transfer coefficients in packed beds. Chem. Eng. Sci. 33, 1375-1384.

Yagi, S., Wakao, N., 1959. Heat and mass transfer from wall to fluid in packed beds. AIChE J 5 (1), 79-85.

Zhang, J., Teixeira, A.R., Jensen, K.F., 2017. Automated measurements of gas-liquid mass transfer in micropacked bed reactors. AIChE J. 64 (2), 564-570. https://doi. org/10.1002/aic.15941. 